\title{
Cohomology of automorphism groups of free groups with twisted coefficients
}

\author{
Oscar Randal-Williams ${ }^{1}$
}

(C) The Author(s) 2017. This article is published with open access at Springerlink.com

\begin{abstract}
We compute the groups $H^{*}\left(\operatorname{Aut}\left(F_{n}\right) ; M\right)$ and $H^{*}\left(\operatorname{Out}\left(F_{n}\right) ; M\right)$ in a stable range, where $M$ is obtained by applying a Schur functor to $H_{\mathbb{Q}}$ or $H_{\mathbb{Q}}^{*}$, respectively the first rational homology and cohomology of $F_{n}$. The answer may be described in terms of stable multiplicities of irreducibles in the plethysm $\mathrm{Sym}^{k} \circ \mathrm{Sym}^{l}$ of symmetric powers. We also compute the stable integral cohomology groups of $\operatorname{Aut}\left(F_{n}\right)$ with coefficients in $H$ or $H^{*}$.
\end{abstract}

Keywords Automorphisms of free groups · Homology stability

Mathematics Subject Classification 20F28 · 20J06 · 57R20

\section{Statement of results}

Galatius [12] has proved the remarkable theorem that the natural homomorphisms

$$
\Sigma_{n} \longrightarrow \operatorname{Aut}\left(F_{n}\right) \longrightarrow \operatorname{Out}\left(F_{n}\right)
$$

both induce homology isomorphisms in degrees $2 * \leq n-3$ with integral coefficients. His approach is to model $B \operatorname{Out}\left(F_{n}\right)$ as the space $\mathcal{G}_{n}$ of graphs of the homotopy type of $\vee^{n} S^{1}$, and $B \operatorname{Aut}\left(F_{n}\right)$ as the space $\mathcal{G}_{n}^{1}$ of pointed graphs of the same homotopy type. He then produces a natural map from such spaces of graphs to the infinite loop space

Supported by ERC Advanced Grant No. 228082, the Danish National Research Foundation through the Centre for Symmetry and Deformation, and EPSRC Grant EP/M027783/1.

$凶 \quad$ Oscar Randal-Williams

o.randal-williams@dpmms.cam.ac.uk

1 Centre for Mathematical Sciences, Wilberforce Road, Cambridge CB3 0WB, UK 
$Q_{0}\left(S^{0}\right)$, which he shows has a certain homological connectivity. One consequence of this is that $H^{i}\left(\operatorname{Aut}\left(F_{n}\right) ; \mathbb{Q}\right)=0$ for $0<i \leq \frac{n-3}{2}$.

At the same time, Satoh [26,27] has studied the low dimensional (co)homology of $\operatorname{Aut}\left(F_{n}\right)$ and $\operatorname{Out}\left(F_{n}\right)$ with coefficients in the module $H:=H_{1}\left(F_{n} ; \mathbb{Z}\right)$ given by the abelianisation of $F_{n}$, and in the dual module $H^{*}:=H^{1}\left(F_{n} ; \mathbb{Z}\right)$. His methods are those of combinatorial group theory, and proceed by calculation with a presentation of these groups.

Our goal is to show that the stable cohomology of $\operatorname{Aut}\left(F_{n}\right)$ and $\operatorname{Out}\left(F_{n}\right)$ with twisted coefficients may also be approached with the geometric techniques used by Galatius, along with a little representation theory. The method we shall introduce is quite general and may be applied whenever a suitable Madsen-Weiss-type theorem has been proved; in "Appendix B" we will show how to use it to recover theorems of Looijenga [20] and Kawazumi [19] on the stable cohomology of mapping class groups with twisted coefficients.

For $\operatorname{Aut}\left(F_{n}\right)$ and $\operatorname{Out}\left(F_{n}\right)$ we will consider cohomology with coefficients in the modules $H_{\mathbb{Q}}^{*}:=H^{1}\left(F_{n} ; \mathbb{Q}\right)$ and $H_{\mathbb{Q}}:=H_{1}\left(F_{n} ; \mathbb{Q}\right)$, and more generally with coefficients in $S_{\lambda}\left(H_{\mathbb{Q}}^{*}\right)$ and $S_{\lambda}\left(H_{\mathbb{Q}}\right)$, where $S_{\lambda}(-)$ is the Schur functor associated to a partition $\lambda \vdash q$, which we think of as being given by a Young diagram. To define this, recall that to such a partition there is an associated irreducible $\mathbb{Q}\left[\Sigma_{q}\right]$-module $S^{\lambda}$, the Specht module. For a $\mathbb{Q}$-vector space $V$ we may consider $V^{\otimes q}$ as a $\mathbb{Q}\left[\Sigma_{q}\right]$-module by permuting the factors, and we may hence form $S_{\lambda}(V):=\operatorname{Hom}_{\Sigma_{q}}\left(S^{\lambda}, V^{\otimes q}\right)$. This construction defines the Schur functor $S_{\lambda}$. It is a basic result that $S_{\lambda}(V)$ is an irreducible representation of $G L(V)$.

In fact, our result is best expressed as calculating $H^{*}\left(\operatorname{Aut}\left(F_{n}\right) ; H_{\mathbb{Q}}^{\otimes q}\right)$ as a $\mathbb{Q}\left[\Sigma_{q}\right]$ module. The result for $S_{\lambda}\left(H_{\mathbb{Q}}\right)$ may then be extracted as

$$
H^{*}\left(\operatorname{Aut}\left(F_{n}\right) ; S_{\lambda}\left(H_{\mathbb{Q}}\right)\right)=\operatorname{Hom}_{\Sigma_{q}}\left(S^{\lambda}, H^{*}\left(\operatorname{Aut}\left(F_{n}\right) ; H_{\mathbb{Q}}^{\otimes q}\right)\right) .
$$

We write $\mathbb{Q}^{-}$for the sign representation of $\Sigma_{q}$.

\section{Theorem A}

(i) $H^{*}\left(\operatorname{Aut}\left(F_{n}\right) ;\left(H^{*}\right)^{\otimes q}\right)=0$ for $2 * \leq n-q-3$.

(ii) $H^{*}\left(\operatorname{Aut}\left(F_{n}\right) ; H_{\mathbb{Q}}^{\otimes q}\right)=0$ for $2 * \leq n-q-3$ if $* \neq q$, and $H^{q}\left(\operatorname{Aut}\left(F_{n}\right) ; H_{\mathbb{Q}}^{\otimes q}\right) \otimes$ $\mathbb{Q}^{-}$is the permutation module on the set of partitions of $\{1,2, \ldots, q\}$.

Theorem A (i) may also be deduced from work of Djament-Vespa [9]. We believe that Theorem A (ii) may also be obtained by combining work of Djament [8] and Vespa [28].

However, more important than these particular results is our technique, which is of very general applicability. For example, it can easily be modified to obtain results for $\operatorname{Out}\left(F_{n}\right)$.

\section{Theorem B}

(i) $H^{*}\left(\operatorname{Out}\left(F_{n}\right) ;\left(H_{\mathbb{Q}}^{*}\right)^{\otimes q}\right)=0$ for $2 * \leq n-q-3$.

(ii) $H^{*}\left(\operatorname{Out}\left(F_{n}\right) ; H_{\mathbb{Q}}^{\otimes q}\right)=0$ for $2 * \leq n-q-3$ if $* \neq q$, and as long as $n \geq 4 q+3$ $H^{q}\left(\operatorname{Out}\left(F_{n}\right) ; H_{\mathbb{Q}}^{\otimes q}\right) \otimes \mathbb{Q}^{-}$is the permutation module on the set of partitions of $\{1,2, \ldots, q\}$ having no parts of size 1 . 
We give tables listing the dimensions of the groups $H^{|\lambda|}\left(\operatorname{Aut}\left(F_{n}\right) ; S_{\lambda}\left(H_{\mathbb{Q}}\right)\right)$ and $H^{|\lambda|}\left(\operatorname{Out}\left(F_{n}\right) ; S_{\lambda}\left(H_{\mathbb{Q}}\right)\right)$ for $|\lambda| \leq 6$ in "Appendix C".

Each partition of $\{1,2, \ldots, q\}$ may be expressed as a partition of a smaller set with no parts of size 1 along with the set of parts of size 1, which translates to the expression

$$
H^{q}\left(\operatorname{Aut}\left(F_{n}\right) ; H_{\mathbb{Q}}^{\otimes q}\right) \cong \bigoplus_{\ell \leq q} \operatorname{Ind}_{\Sigma_{\ell} \times \Sigma_{q-\ell}}^{\Sigma_{q}}\left(H^{\ell}\left(\operatorname{Out}\left(F_{n}\right) ; H_{\mathbb{Q}}^{\otimes \ell}\right) \otimes \mathbb{Q}^{-}\right)
$$

as long as $n \geq 4 q+3$. Applying $\operatorname{Hom}_{\Sigma_{q}}\left(S^{\lambda},-\right)$ and using the Pieri rule gives the pleasant formula

$$
H^{|\lambda|}\left(\operatorname{Aut}\left(F_{n}\right) ; S_{\lambda}\left(H_{\mathbb{Q}}\right)\right) \cong \bigoplus_{\mu \in \rho(\lambda)} H^{|\mu|}\left(\operatorname{Out}\left(F_{n}\right) ; S_{\mu}\left(H_{\mathbb{Q}}\right)\right)
$$

as long as $n \geq 4|\lambda|+3$, where $\rho(\lambda)$ denotes the set of Young diagrams which may be obtained from $\lambda$ by removing at most one box from each row.

\subsection{Stable plethysm of symmetric powers}

For a Young diagram $\mu$ and integers $k$ and $l$ such that $2|\mu| \leq k l$, let $(k l-|\mu|, \mu)$ be the Young diagram obtained by adding a row of length $k l-|\mu|$ to the top of $\mu$, and let $v^{k, l}(\mu)$ denote the multiplicity of the irreducible $G L(V)$-representation $S_{(k l-|\mu|, \mu)}(V)$ in $\operatorname{Sym}^{k}\left(\operatorname{Sym}^{l}(V)\right)$. Manivel has shown [21] that the numbers $\nu^{k, l}(\mu)$ are increasing and eventually constant functions of both $k$ and $l$, and we write $v^{\infty}(\mu)$ for the stable value. This stable value is attained as soon as $l \geq \mu_{1}$ and $k \geq|\mu|$. Using the work of Manivel, we are able to relate these stable multiplicities directly to the cohomology of $\operatorname{Out}\left(F_{n}\right)$, as follows.

Theorem C As long as $n \geq 4|\lambda|+3$ we have

$$
\operatorname{dim}_{\mathbb{Q}} H^{|\lambda|}\left(\operatorname{Out}\left(F_{n}\right) ; S_{\lambda}\left(H_{\mathbb{Q}}\right)\right)=v^{\infty}\left(\lambda^{\prime}\right)
$$

and as long as $n \geq 2|\lambda|+3$ we have

$$
\operatorname{dim}_{\mathbb{Q}} H^{|\lambda|}\left(\operatorname{Aut}\left(F_{n}\right) ; S_{\lambda}\left(H_{\mathbb{Q}}\right)\right)=\sum_{\mu \in \rho(\lambda)} v^{\infty}\left(\mu^{\prime}\right)
$$

It follows directly from [21, Proposition 4.4.1] that $H^{|\lambda|}\left(\operatorname{Out}\left(F_{\infty}\right) ; S_{\lambda}\left(H_{\mathbb{Q}}\right)\right)=0$ if $2 \lambda_{1}>|\lambda|$, or if $2 \lambda_{1}=|\lambda|$ and $\lambda$ does not consist of two rows of equal length.

\subsection{Symmetric and exterior powers}

In order to demonstrate how our theorems may be used, we compute the dimension of the associated cohomology groups for the modules $\wedge^{q}\left(H_{\mathbb{Q}}\right)$ and $\operatorname{Sym}^{q}\left(H_{\mathbb{Q}}\right)$. 


\section{Corollary D}

(i) For $n \geq 2 q+3, H^{q}\left(\operatorname{Aut}\left(F_{n}\right) ; \wedge^{q}\left(H_{\mathbb{Q}}\right)\right)$ has dimension given by the number of partitions of $q$; for $n \geq 4 q+3, H^{q}\left(\operatorname{Out}\left(F_{n}\right) ; \wedge^{q}\left(H_{\mathbb{Q}}\right)\right)$ has dimension given by the number of partitions of $q$ into pieces none of which are 1 .

(ii) Let $q \geq 2$. For $n \geq 2 i+q+3$,

$$
H^{i}\left(\operatorname{Aut}\left(F_{n}\right) ; \operatorname{Sym}^{q}\left(H_{\mathbb{Q}}\right)\right)=H^{i}\left(\operatorname{Out}\left(F_{n}\right) ; \operatorname{Sym}^{q}\left(H_{\mathbb{Q}}\right)\right)=0 .
$$

Proof The dimension of $H^{q}\left(\operatorname{Aut}\left(F_{n}\right) ; \wedge^{q}\left(H_{\mathbb{Q}}\right)\right)$ is the multiplicity of the sign representation in $H^{q}\left(\operatorname{Aut}\left(F_{n}\right) ; H_{\mathbb{Q}}^{\otimes q}\right)$, which by Theorem A is the multiplicity of the trivial representation in the permutation module for the set of partitions of $\{1,2, \ldots, q\}$ : this is the number of partitions of $q$.

The dimension of $H^{q}\left(\operatorname{Aut}\left(F_{n}\right) ; \operatorname{Sym}^{q}\left(H_{\mathbb{Q}}\right)\right)$ is the multiplicity of the trivial representation in $H^{q}\left(\operatorname{Aut}\left(F_{n}\right) ; H_{\mathbb{Q}}^{\otimes q}\right)$, which by Theorem A is the multiplicity of the sign representation in the permutation module for the set of partitions of $\{1,2, \ldots, q\}$. This may be computed via inner product of characters as $\frac{1}{q !}$ times

$$
\sum_{\sigma \in \Sigma_{q}} \#\{\text { partitions } P \text { such that } \sigma(P)=P\} \cdot \operatorname{sign}(\sigma)=\sum_{\substack{\text { partitions } \\ P}} \sum_{\substack{\sigma \in \Sigma_{q} \\ \sigma(P)=P}} \operatorname{sign}(\sigma),
$$

but, writing $\Sigma_{P} \leq \Sigma_{q}$ for the stabiliser of a partition $P$, we have that $\sum_{\sigma \in \Sigma_{P}} \operatorname{sign}(\sigma)$ is $\left|\Sigma_{P}\right|$ times the multiplicity of the sign representation in the trivial representation of $\Sigma_{P}$, which is zero (as $\Sigma_{P}$ always contains at least one transposition if $q \geq 2$ ).

The arguments for $\operatorname{Out}\left(F_{n}\right)$ are identical.

\subsection{Integral and torsion results}

Finally, our technique can be made to give integral and local information as well. It is not hard to show that $H^{i}\left(\operatorname{Aut}\left(F_{n}\right) ; H^{*}\right)=0$ for $2 i \leq n-4$ (see Proposition 2.3), but we also have the following.

\section{Theorem E}

(i) $H^{*}\left(\operatorname{Aut}\left(F_{\infty}\right) ; H\right)$ is a free $H^{*}\left(\operatorname{Aut}\left(F_{\infty}\right) ; \mathbb{Z}\right)$-module (on a single generator in degree 1).

(ii) For a partition $\lambda \vdash q$ and a prime number $p>q, H^{*}\left(\operatorname{Aut}\left(F_{\infty}\right) ; S_{\lambda}\left(H \otimes \mathbb{Z}_{(p)}\right)\right)$ is a free $H^{*}\left(\operatorname{Aut}\left(F_{\infty}\right) ; \mathbb{Z}_{(p)}\right)$-module (on generators in degree $q$, the number of which may be deduced from Theorem A).

That Theorem E (ii) might hold was suggested to the author by Aurélien Djament upon hearing of Theorem E (i). We refer to Sect. 6 for a description of what we mean by the Schur functor $S_{\lambda}$ in the $p$-local setting.

One may deduce from Theorem $\mathrm{E}$ (i) that $H^{i}\left(\operatorname{Out}\left(F_{n}\right) ; H \otimes \mathbb{Z}\left[\frac{1}{n-1}\right]\right)=0$ for $2 i \leq$ $n-4$. In "Appendix A" we describe how the calculation $H^{1}\left(\operatorname{Out}\left(F_{n}\right) ; H\right) \cong \mathbb{Z} /(n-1)$ for $n \geq 9$ follows from a reasonable-seeming conjecture about spaces of graphs. 


\section{An observation regarding homology stability}

The observations of this section are no doubt known to some experts. The groups $\operatorname{Aut}\left(F_{n}\right)$ and $\operatorname{Out}\left(F_{n}\right)$ fit into a more general family of groups denoted $\Gamma_{n, s}$ by HatcherVogtmann [16], where $\operatorname{Aut}\left(F_{n}\right)=\Gamma_{n, 1}$ and $\operatorname{Out}\left(F_{n}\right)=\Gamma_{n, 0}$. Classifying spaces for these may be taken to be the spaces $\mathcal{G}_{n}^{s}$ of graphs of the homotopy type of $\vee^{n} S^{1}$ equipped with $s$ distinct ordered marked points.

Hatcher and Vogtmann [16] prove that the map $\mathcal{G}_{n}^{s} \rightarrow \mathcal{G}_{n+1}^{s}$ (defined for $s>0$ ) that adds a loop at a particular marked point induces an integral homology isomorphism in degrees $2 * \leq n-2$ (and induces a rational homology isomorphism in degrees $5 * \leq 4 n-10)$. Furthermore, the map $\mathcal{G}_{n}^{s} \rightarrow \mathcal{G}_{n}^{s-1}$ that forgets a marked point is an integral homology isomorphism in degrees $2 * \leq n-3$ (or $2 * \leq n-4$ if it is the last marked point).

We can make an immediate observation regarding cohomology with coefficients in $H^{*}$ from this homology stability result. There is an extension $F_{n} \rightarrow \operatorname{Aut}\left(F_{n}\right) \rightarrow$ $\operatorname{Out}\left(F_{n}\right)$, and the corresponding Leray-Hochschild-Serre spectral sequence has two rows. However, as the projection is a homology equivalence in a range of degrees we deduce

Proposition 2.1 The groups $H^{*}\left(\operatorname{Out}\left(F_{n}\right) ; H^{*}\right)$ are zero for $2 * \leq n-6$.

Similarly, there is a fibration with section $\vee^{n} S^{1} \rightarrow \mathcal{G}_{n}^{2} \rightarrow \mathcal{G}_{n}^{1}$ and the projection map is a homology equivalence in a range of degrees, so

Proposition 2.2 The groups $H^{*}\left(\operatorname{Aut}\left(F_{n}\right) ; H^{*}\right)$ are zero for $2 * \leq n-4$.

More generally, the map $\mathcal{G}_{n}^{k+1} \rightarrow \mathcal{G}_{n}^{1}$ has fibre $\left(\vee^{n} S^{1}\right)^{k}$ and $\operatorname{Aut}\left(F_{n}\right)$ acts on its homology diagonally. Thus for $k=2$ the Serre spectral sequence has three rows, with

$$
E_{2}^{*, 1}=H^{*}\left(\operatorname{Aut}\left(F_{n}\right) ; H^{*} \oplus H^{*}\right)=0 \text { for } 2 * \leq n-4 \text { by Proposition } 2.2
$$

and

$$
E_{2}^{*, 2}=H^{*}\left(\operatorname{Aut}\left(F_{n}\right) ; H^{*} \otimes H^{*}\right) .
$$

Using the fact that the projection map is a homology equivalence in degrees $2 * \leq n-2$, we deduce that $H^{*}\left(\operatorname{Aut}\left(F_{n}\right) ; H^{*} \otimes H^{*}\right)=0$ for $2 * \leq n-8$. Continuing in this way for higher $k$, we establish the following proposition.

Proposition 2.3 For all $q \geq 1, H^{*}\left(\operatorname{Aut}\left(F_{n}\right) ;\left(H^{*}\right)^{\otimes q}\right)$ is zero for $2 * \leq n-4 q$.

\section{Homology stability with coefficient systems}

By Galatius' theorem, the groups Aut $\left(F_{n}\right)$ are closely related to the symmetric groups, but also share many properties with mapping class groups of surfaces. These three families of groups are known to exhibit homological stability for integral homology, but symmetric groups and mapping class groups also exhibit homological stability for certain systems of coefficients, those of "finite degree", a notion that is originally 
due to Dwyer [10] in his study of homological stability for general linear groups with coefficient systems.

This notion of degree may be formalised, in the context of free groups, as follows.

Definition 3.1 Let $\mathfrak{G r}$ denote the category whose objects are the finitely generated free groups, and where a morphism from $G$ to $H$ is given by a pair

$$
(f: G \rightarrow H, X \leq H)
$$

consisting of an injective group homomorphism $f$ and a finitely-generated (free) subgroup $X \leq H$ such that $H=f(G) * X$.

A coefficient system is a covariant functor $V: \mathfrak{G r} \rightarrow \mathbf{A b}$ to the category of abelian groups. We declare the constant functors to be polynomial of degree 0 , and more generally we declare a functor $V$ to be polynomial of degree $\leq k$ if

(i) $V$ sends the the canonical morphism $s_{G}:=($ inc $: G \rightarrow G * \mathbb{Z}, \mathbb{Z}$ ) to an injection, and

(ii) the new coefficient system $G \mapsto \operatorname{Coker}\left(s_{G}: V(G) \rightarrow V(G * \mathbb{Z})\right)$ is polynomial of degree $\leq k-1$.

In a 2010 preprint [23], we conjectured (based on the analogy with general linear groups [10] and mapping class groups [17]) that the groups $H_{*}\left(\operatorname{Aut}\left(F_{n}\right) ; V\left(F_{n}\right)\right)$ should exhibit homological stability in degrees $2 * \leq n-k-2$ when $V$ is a polynomial coefficient system of degree $\leq k$. Since then, in joint work with Wahl [25] we have established a quite general homological stability theorem with polynomial coefficients, and using the highly-connected simplicial complexes of [15] it applies in this case. The result obtained is as follows.

Theorem 3.2 (Randal-Williams-Wahl [25]) If $V$ is a polynomial coefficient system of degree $\leq k$, then the natural map

$$
H_{*}\left(\operatorname{Aut}\left(F_{n}\right) ; V\left(F_{n}\right)\right) \longrightarrow H_{*}\left(\operatorname{Aut}\left(F_{n+1}\right) ; V\left(F_{n+1}\right)\right)
$$

induces an epimorphism for $2 * \leq n-k-1$ and an isomorphism for $2 * \leq n-k-3$.

One advantage of the category $\mathfrak{G} \mathfrak{r}$ over the more naïve category $\mathfrak{g r}$ of finitely generated free groups and injective homomorphisms is that there are more functors out of it.

\section{Definition 3.3}

(i) Let $H: \mathfrak{G r} \rightarrow \mathbf{A b}$ be the coefficient system sending $G$ to $H_{1}(G ; \mathbb{Z})=G^{a b}$, and sending a morphism $(f: G \rightarrow H, X)$ to $f_{*}$. There is an exact sequence

$$
0 \longrightarrow H(G) \stackrel{H\left(s_{G}\right)}{\longrightarrow} H(G * \mathbb{Z}) \longrightarrow \mathbb{Z} \longrightarrow 0
$$

so this coefficient system is polynomial of degree 1 . 
(ii) Let $H^{*}: \mathfrak{G r} \rightarrow \mathbf{A b}$ be the coefficient system sending $G$ to $H^{1}(G ; \mathbb{Z})=$ $\operatorname{Hom}\left(G^{a b}, \mathbb{Z}\right)$, and sending a morphism $(f: G \rightarrow H, X)$ to the linear dual of

$$
H_{1}(H ; \mathbb{Z})=H_{1}(f(G) * X ; \mathbb{Z}) \longrightarrow H_{1}(f(G) ; \mathbb{Z}) \cong H_{1}(G ; \mathbb{Z})
$$

(Note that this does not define a functor on $\mathfrak{g r} !)$ There is an exact sequence

$$
0 \longrightarrow H^{*}(G) \stackrel{H^{*}\left(s_{G}\right)}{\longrightarrow} H^{*}(G * \mathbb{Z}) \longrightarrow \mathbb{Z} \longrightarrow 0
$$

so this coefficient system is polynomial of degree 1 .

Remark 3.4 Not only can more coefficient systems be defined on $\mathfrak{G r}$ than on $\mathfrak{g r}$, but it follows from recent work of Djament-Vespa [9] that the most homologically interesting functors must be defined here: they show that if $V$ is a polynomial coefficient system which is reduced (i.e. $V(\{e\})=0$ ) and factors through $\mathfrak{g r}$, then

$$
\underset{n \rightarrow \infty}{\operatorname{colim}} H_{*}\left(\operatorname{Aut}\left(F_{n}\right) ; V\left(F_{n}\right)\right)=0 .
$$

The coefficient system $H^{*}$ is reduced, but does not factor through $\mathfrak{g r}$ : indeed, Satoh [26] has shown that $\operatorname{colim}_{n \rightarrow \infty} H_{1}\left(\operatorname{Aut}\left(F_{n}\right) ; H^{*}\right)=\mathbb{Z}$, and we will show how to recover (the dual version of) this in Sect. 6.

There are some easy consequences of the definition of polynomiality which allow us to compute or estimate degrees of coefficient systems. Firstly, a summand of a polynomial functor of degree $\leq k$ is again polynomial of degree $\leq k$, and an extension of two polynomial functors of degree $\leq k$ is again polynomial of degree $\leq k$. Secondly, if $V$ and $W$ are coefficient systems which are polynomial of degree $k$ and $\ell$ respectively, and if they both take values in flat $\mathbb{Z}$-modules, then $V \otimes_{\mathbb{Z}} W$ is polynomial of degree $\leq(k+\ell)$. More generally, if $\mathbb{F}$ is a field and $V, W: \mathfrak{G r} \rightarrow \mathbb{F}$-mod $\rightarrow \mathbf{A b}$ are coefficient systems which are polynomial of degree $k$ and $\ell$ respectively and factor through the category of $\mathbb{F}$-modules, then $V \otimes_{\mathbb{F}} W$ is polynomial of degree $\leq(k+\ell)$.

We will often be interested in cohomology rather than homology, for which we will use the following result of Universal Coefficient-type, which is surely standard but for which we could not find a reference.

Lemma 3.5 Let $G$ be a group, $R$ be a PID, and $M$ a left $R[G]$-module, and write $M^{*}=\operatorname{Hom}_{R}(M, R)$, a right $R[G]$-module. There is a natural short exact sequence

$0 \longrightarrow \operatorname{Ext}_{R}^{1}\left(H_{i-1}(G ; M), R\right) \longrightarrow H^{i}\left(G ; M^{*}\right) \longrightarrow \operatorname{Hom}_{R}\left(H_{i}(G ; M), R\right) \longrightarrow 0$.

Proof Analogous to [3, Proposition 7.1]. Let $P_{\bullet} \rightarrow R$ be a projective right $R[G]$ module resolution, so $C^{\bullet}:=\operatorname{Hom}_{R[G]}\left(P_{\bullet}, M^{*}\right)$ is a cochain complex of $R$-modules which computes $H^{*}\left(G ; M^{*}\right)$. Writing

$$
C^{\bullet}=\operatorname{Hom}_{R}\left(P_{\bullet}, M^{*}\right)^{G} \cong \operatorname{Hom}_{R}\left(P_{\bullet} \otimes_{R} M, R\right)^{G} \cong \operatorname{Hom}_{R}\left(P_{\bullet} \otimes_{R[G]} M, R\right)
$$


and applying the Universal Coefficient Theorem (e.g. [4, p. 114]), using the fact that a submodule of a free module over a PID is free, gives the desired sequence.

Using the stability theorem and Lemma 3.5, we may improve Proposition 2.3 to the following.

Corollary 3.6 For all $q \geq 1, H^{*}\left(\operatorname{Aut}\left(F_{n}\right) ;\left(H^{*}\right)^{\otimes q}\right)=0$ for $2 * \leq n-q-3$.

This proves Theorem A (i). We remark that Theorem A (i) also follows from [9, Théorème 1] and Lemma 3.5, because the functor $H^{\otimes q}: \mathfrak{G r} \rightarrow \mathfrak{g r} \rightarrow \mathbf{A b}$ is polynomial and vanishes on the trivial group (for $q \geq 1$ ), so by that theorem $H_{*}\left(\operatorname{Aut}\left(F_{n}\right) ; H^{\otimes q}\right)$ vanishes in the stable range.

Finally, we record the stability range for cohomology with coefficients in $S_{\lambda}\left(H_{\mathbb{Q}}\right)$ with $\lambda \vdash q$. This follows from $S_{\lambda}\left(H_{\mathbb{Q}}\right) \cong S_{\lambda}\left(H_{\mathbb{Q}}^{*}\right)^{*}$, that $S_{\lambda}\left(H_{\mathbb{Q}}^{*}\right)$ is polynomial of degree $\leq q$ as it is a summand of $\left(H_{\mathbb{Q}}^{*}\right)^{\otimes q}$, the stability theorem, and Lemma 3.5.

Corollary 3.7 The groups $H^{i}\left(\operatorname{Aut}\left(F_{n}\right) ; S_{\lambda}\left(H_{\mathbb{Q}}\right)\right)$ are independent of $n$ for $2 i \leq n-$ $q-3$.

\section{Graphs labeled by a space $X$}

Let $\left(X, x_{0}\right)$ be a based space. Let us write $\mathcal{G}^{X}$ for the topological category whose objects are finite sets inside $\mathbb{R}^{\infty}$, and whose morphisms from $S \subset \mathbb{R}^{\infty}$ to $T \subset \mathbb{R}^{\infty}$ consist of a real number $t>0$ and a graph $\Gamma \subset[0, t] \times \mathbb{R}^{\infty}$ with leaves $\{0\} \times S$ and $\{t\} \times T$, equipped with a continuous map $f:(\Gamma, S \cup T) \rightarrow\left(X, x_{0}\right)$. This may be given a topology following [12]. Choosing once and for all an embedding $\{1,2, \ldots, s\} \subset \mathbb{R}^{\infty}$ the space $\mathcal{G}_{n}^{s}(X)$ may be defined as the subspace of $\mathcal{G}^{X}(\emptyset,\{1,2, \ldots, s\})$ consisting of those graphs which are connected and homotopy equivalent to $\vee^{n} S^{1}$.

Let $\mathcal{G}_{n}^{s}(X)_{*} \subset \mathcal{G}_{n}^{s}(X) \times \mathbb{R}^{\infty+1}$ be the subspace given by tuples $(t, \Gamma, f ; p)$ where $p \in \Gamma$. Forgetting the point $p$ gives a map

$$
\pi: \mathcal{G}_{n}^{s}(X)_{*} \longrightarrow \mathcal{G}_{n}^{s}(X)
$$

which is a fibration with fibre over the point $(t, \Gamma, f) \in \mathcal{G}_{n}^{s}(X)$ given by the graph $\Gamma$. Furthermore, sending $(t, \Gamma, f, p)$ to $f(p) \in X$ defines a map

$$
e: \mathcal{G}_{n}^{s}(X)_{*} \longrightarrow X .
$$

As the map $\pi$ is a fibration with homotopy-finite fibres, it admits a Becker-Gottlieb transfer map $\operatorname{trf}_{\pi}: \Sigma_{+}^{\infty} \mathcal{G}_{n}^{s}(X) \rightarrow \Sigma_{+}^{\infty} \mathcal{G}_{n}^{s}(X)_{*}$. Composing this with $\Sigma_{+}^{\infty} e$ and taking the adjoint gives a map

$$
\tau_{n}^{s}: \mathcal{G}_{n}^{s}(X) \longrightarrow Q_{1-n}\left(X_{+}\right)
$$

to the free infinite loop space on $X$, landing in the path component indexed by $1-n$, the Euler characteristic of $\vee^{n} S^{1}$. This map is natural in $X$, and factors through the $\operatorname{map} \tau_{n}^{0}: \mathcal{G}_{n}(X) \rightarrow Q_{1-n}\left(X_{+}\right)$up to homotopy. 


\section{Theorem 4.1 The induced map}

$$
\tau_{\infty}^{1}: \underset{n \rightarrow \infty}{\operatorname{hocolim}} \mathcal{G}_{n}^{1}(X) \longrightarrow Q_{0}\left(X_{+}\right)
$$

is an integral homology equivalence as long as $X$ is path-connected.

The point of this theorem is that the right-hand side may be computed: the rational cohomology of $Q_{1-n}\left(X_{+}\right)$may be described compactly as $S^{*}\left(\widetilde{H}^{*}(X ; \mathbb{Q})\right)$, the free graded-commutative algebra on the reduced cohomology of $X$.

Proof sketch Following [12], and keeping track of the role that the maps to $X$ play, one may show that $B \mathcal{G}^{X} \simeq Q\left(S^{1} \wedge X_{+}\right)$. Furthermore, under this equivalence the composition

$$
\mathcal{G}_{n}(X) \subset \mathcal{G}^{X}(\emptyset, \emptyset) \longrightarrow \Omega B \mathcal{G}^{X} \simeq Q\left(X_{+}\right)
$$

is weakly homotopic to $\tau_{n}^{0}$ by the analogue (with maps to $X$ ) of the discussion in Section 5.3 of [12].

Now we claim that the natural map

$$
\underset{n \rightarrow \infty}{\operatorname{hocolim}} \mathcal{G}_{n}^{1}(X) \longrightarrow \Omega_{[\emptyset,\{0\}]} B \mathcal{G}^{X}
$$

is an integral homology equivalence as long as $X$ is path-connected. This may be proved following [12] and also using ideas from [13]. The crucial point is to consider the category $\mathcal{G}_{\bullet}^{X}$ where objects $S$ contain the origin $0 \in \mathbb{R}^{\infty}$ and morphisms $\Gamma$ contain the interval $[0, t] \times\{0\}$ on which the function $f$ is constant. The inclusion $\mathcal{G}_{\bullet}^{X} \rightarrow \mathcal{G}^{X}$ may be seen to induce an equivalence on classifying spaces as in [13, Lemma 4.6]. One then considers the subcategory $\mathcal{G}_{\bullet, c}^{X} \subset \mathcal{G}_{\bullet}^{X}$ having only object $\{0\}$ and in which the morphisms are required to be connected. We claim that this inclusion induces an equivalence on classifying spaces. To make morphisms connected use a move similar to that of [12, Lemma 4.24] which connects an arbitrary path component to the standard stick $\mathbb{R} \times\{0\} \subset \mathbb{R} \times \mathbb{R}^{\infty}$ (this requires $X$ to be path-connected). To reduce to a single object use a move similar to that of [13, Section 4] to make objects consist of a single point, then isotope them into standard position as in [13, Proposition 4.26].

The category $\mathcal{G}_{\bullet, c}^{X}$ is therefore a monoid, and, as we can slide edges along the standard interval $[0, t] \times\{0\}$, it is a homotopy commutative monoid. We may thus apply the group-completion theorem to it, showing that

$$
\operatorname{hocolim} \mathcal{G}_{\bullet, c}^{X}(\{0\},\{0\}) \longrightarrow \Omega B \mathcal{G}_{\bullet, c}^{X},
$$

is a homology isomorphism, where the homotopy colimit is formed by left multiplication with a connected graph of genus 1 . By precomposing with a morphism $\emptyset \rightsquigarrow\{0\} \in \mathcal{G}^{X}$ given by an interval, this map is easily compared with that in the statement of the theorem. 
The main technical result we will require is the following homological stability theorem for the spaces $\mathcal{G}_{n}^{s}(X)$. We will deduce it from (two) arguments of CohenMadsen in the analogous situation of surfaces with maps to a background space.

Theorem 4.2 Suppose that $X$ is simply-connected.

(i) The map $\mathcal{G}_{n}^{1}(X) \rightarrow \mathcal{G}_{n+1}^{1}(X)$ induces a homology isomorphism in degrees $2 * \leq$ $n-3$.

(ii) The map $\mathcal{G}_{n}^{1}(X) \rightarrow \mathcal{G}_{n}(X)$ induces an isomorphism on homology with $\mathbb{Z}\left[\frac{1}{n-1}\right]$ module coefficients in degrees $2 * \leq n-3$.

Proof For part (i) we follow the argument of Cohen and Madsen [6]. There is a map of homotopy fibre sequences

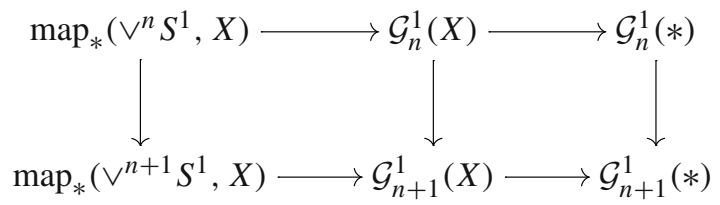

so it is enough to show that $\operatorname{Aut}\left(F_{n}\right) \circlearrowleft H_{i}\left(\operatorname{map}_{*}\left(\vee^{n} S^{1}, X\right) ; \mathbb{Z}\right)$ is part of a polynomial coefficient system of degree $\leq i$. In this case the map of Serre spectral sequences will induce an isomorphism on $E_{s, t}^{2}$ for $2 s \leq n-t-3$ and an epimorphism for $2 s \leq n-t-1$; in particular it induces an isomorphism for $2(s+t) \leq n-3$ and an epimorphism for $2(s+t) \leq n-1$. It follows from the spectral sequence comparison theorem that the map $H_{*}\left(\mathcal{G}_{n}^{1}(X)\right) \rightarrow H_{*}\left(\mathcal{G}_{n+1}^{1}(X)\right)$ in an isomorphism in degrees $2 * \leq n-3$ and an epimorphism in degrees $2 * \leq n-1$.

We define a coefficient system $V_{i}^{X}: \mathfrak{G r} \rightarrow \mathbf{A b}$ on objects by

$$
V_{i}^{X}(G):=H_{i}\left(\operatorname{map}_{*}(B G, X) ; \mathbb{Z}\right)
$$

and on a morphism $(f: G \rightarrow H, X)$ we use precomposition by

$$
B H=B(f(G) * X) \longrightarrow B(f(G)) \cong B G
$$

This defines a coefficient system, and since $B(G * \mathbb{Z}) \rightarrow B G$ is split surjective the stabilisation maps $V_{i}^{X}\left(s_{G}\right)$ are all split injective. The coefficient system $V_{0}^{X}$ agrees with the constant coefficient system $\mathbb{Z}$, as $X$ has been assumed to be simply-connected: in particular it is polynomial of degree 0 .

Let us suppose for an induction that $V_{j}^{X}$ has degree $\leq j$ for all $j<i$. Consider the homotopy fibre sequence

$$
\operatorname{map}_{*}(B G, X) \stackrel{\iota}{\longrightarrow} \operatorname{map}_{*}(B(G * \mathbb{Z}), X) \longrightarrow \operatorname{map}_{*}(B \mathbb{Z}, X),
$$

where the map $\iota$ induces $V_{i}^{X}\left(s_{G}\right)$ on $i$ th homology. As $\iota$ is split injective, the Serre spectral sequence for this fibration (which is over a path-connected base) collapses, 
and we find that $\operatorname{Coker}\left(V_{i}^{X}(G) \rightarrow V_{i}^{X}(G * \mathbb{Z})\right)$ has a filtration with associated graded

$$
\left\{H_{i-j}\left(\Omega X ; V_{j}^{X}(G)\right)\right\}_{j=0}^{i-1}
$$

Each $V_{j}^{X}(-)$ is polynomial of degree $\leq i-1$ so $H_{i-j}\left(\Omega X ; V_{j}^{X}(-)\right)$ is too; as degree is preserved under extensions it follows that $\operatorname{Coker}\left(V_{i}^{X}(G) \rightarrow V_{i}^{X}(G * \mathbb{Z})\right)$ has degree $\leq i-1$, hence $V_{i}^{X}$ has degree $\leq i$.

For part (ii), we follow a different argument of Cohen and Madsen [7]. We have a diagram

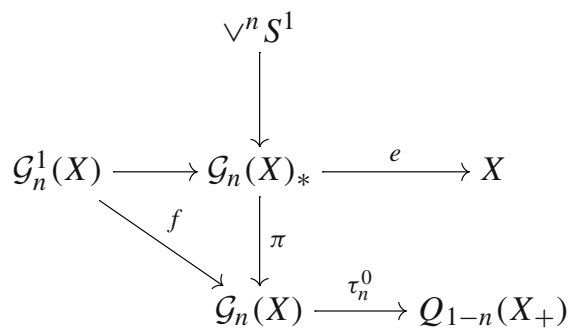

in which the row and column are fibrations, and the map $\tau_{n}^{0}$ is the adjoint to the map of spectra $\Sigma_{+}^{\infty} \mathcal{G}_{n}(X) \stackrel{\operatorname{trf}_{\pi}}{\rightarrow} \Sigma_{+}^{\infty} \mathcal{G}_{n}(X)_{*} \stackrel{e}{\rightarrow} \Sigma_{+}^{\infty} X$ given by the composition of the BeckerGottlieb transfer for the map $\pi$ followed by the map $e$ given by evaluating the map to $X$ at the marked point.

By part (i), Theorem 4.1, and the analogue with maps to $X$ of the discussion in Section 5.3 of [12], the composition $\tau_{n}^{0} \circ f \simeq \tau_{n}^{1}: \mathcal{G}_{n}^{1}(X) \rightarrow Q_{1-n}\left(X_{+}\right)$is an isomorphism in degrees $2 * \leq n-3$, and in particular $\tau_{n}^{0}$ is surjective on homology in this range. It follows from Leray-Hirsch that the map

$$
e \times\left(\tau_{n}^{0} \circ \pi\right): \mathcal{G}_{n}(X)_{*} \longrightarrow X \times Q_{1-n}\left(X_{+}\right)
$$

is also an isomorphism in this range.

Now we have the commutative diagram

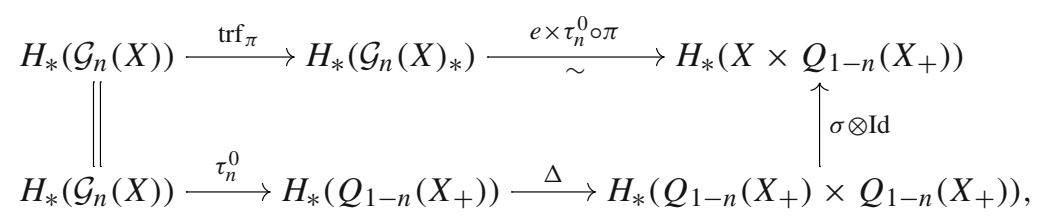

where $\sigma$ denotes the homology suspension. The transfer map $\operatorname{trf}_{\pi}$ is (split) injective with $\mathbb{Z}\left[\frac{1}{n-1}\right]$-module coefficients, so in degrees $2 * \leq n-3$ the map $\tau_{n}^{0}$ is injective too. Hence $\tau_{n}^{0}$ is an isomorphism in this range, so $f$ is too. 


\section{Proof of the main theorems}

\subsection{Recollections on Schur-Weyl duality}

We shall need a small amount of representation theory, but nothing beyond e.g. the first few parts of Chapter 9 of [22]. Recall that for a vector space $V$ (over $\mathbb{Q}$ ) and a partition $\lambda \vdash q$ we have defined $S_{\lambda}(V)$ as $\operatorname{Hom}_{\Sigma_{q}}\left(S^{\lambda}, V^{\otimes q}\right)$. If $V$ is finite-dimensional then as the Specht modules $S^{\lambda}$ give a complete set of irreducible representations of $\Sigma_{q}$ we may write

$$
V^{\otimes q} \cong \bigoplus_{\lambda \vdash q} S_{\lambda}(V) \otimes S^{\lambda}
$$

as $G L(V) \times \Sigma_{q}$-modules. The $S_{\lambda}(V)$ are non-zero as long as $\operatorname{dim}_{\mathbb{Q}} V \geq q$, in which case they are irreducible $G L(V)$-modules.

\subsection{Labeled partitions}

Let us fix a sequence of $\mathbb{Q}$-vector spaces $W_{1}, W_{2}, \ldots$, and consider

$$
F(V):=\operatorname{Sym}^{*}\left(W_{1} \otimes \operatorname{Sym}^{1}(V) \oplus W_{2} \otimes \operatorname{Sym}^{2}(V) \oplus W_{3} \otimes \operatorname{Sym}^{3}(V) \oplus \cdots\right) .
$$

If we consider this as a functor landing in graded vector spaces, by letting $V$ have degree 1, then the homogenous pieces of $F$ are each polynomial functors of $V$ (see [22, §9.7] for this notion). Thus the $\Sigma_{q}$-module $\operatorname{Hom}_{G L(V)}\left(V^{\otimes q}, F(V)\right)$ is independent of $V$ as long as $\operatorname{dim}_{\mathbb{Q}} V \geq q$. We wish to identify this $\Sigma_{q}$-module.

Choose bases $\Omega_{i}$ for the vector spaces $W_{i}$, and let $\mathcal{P}_{q}\left(\Omega_{1}, \Omega_{2}, \ldots\right)$ be the set of partitions $P$ of $\{1,2, \ldots, q\}$ equipped with a labeling of each part $X$ of size $i$ with an element $\ell_{X} \in \Omega_{i}$. For each such datum $(P, \ell)$ there is a map of $G L(V)$-modules

$$
\begin{aligned}
\phi_{P, \ell}: V^{\otimes q} & \longrightarrow \operatorname{Sym}^{*}\left(W_{1} \otimes \operatorname{Sym}^{1}(V) \oplus W_{2} \otimes \operatorname{Sym}^{2}(V) \oplus \cdots\right) \\
v_{1} \otimes \cdots \otimes v_{q} & \longmapsto \prod_{X \subset P}\left[\ell_{X} \cdot \prod_{i \in X} v_{i}\right] .
\end{aligned}
$$

If $\sigma \in \Sigma_{q}$ then $\phi_{P, \ell} \circ \sigma=\phi_{\sigma(P, \ell)}$, giving a $\Sigma_{q}$-equivariant map

$$
\phi: \mathbb{Q}\left\{\mathcal{P}_{q}\left(\Omega_{1}, \Omega_{2}, \ldots\right)\right\} \longrightarrow \operatorname{Hom}_{G L(V)}\left(V^{\otimes q}, F(V)\right)
$$

Proposition 5.1 The map $\phi$ is an isomorphism as long as $\operatorname{dim}_{\mathbb{Q}} V \geq q$.

Proof Let us call an orbit of $\Sigma_{q}$ acting on $\mathcal{P}_{q}\left(\Omega_{1}, \Omega_{2}, \ldots\right)$ a type of partition. It consists of a partition $\lambda \vdash q$ along with an unordered list of labels $L_{i}$ in $\Omega_{i}$ for the parts of size $i$, and we write $\mathcal{P}\left(\lambda ; L_{1}, L_{2}, \ldots\right)$ for this orbit.

The source of $\phi$ splits as a direct sum of (cyclic) modules $\mathbb{Q}\left\{\mathcal{P}\left(\lambda ; L_{1}, L_{2}, \ldots\right)\right\}$ one for each type of partition. For a type of partition $\mathcal{P}\left(\lambda ; L_{1}, L_{2}, \ldots\right)$ and a $\omega \in \Omega_{i}$, let 
us write $|\omega|=i$ and $a_{\omega}$ for the number of parts labeled by $\omega$. The target of $\phi$ splits as a direct sum of terms

$$
M\left(\lambda ; L_{1}, L_{2}, \ldots\right):=\operatorname{Hom}_{G L(V)}\left(V^{\otimes q}, \bigotimes_{\omega \in \cup_{i} \Omega_{i}} \operatorname{Sym}^{a_{\omega}}\left(\{\omega\} \otimes \operatorname{Sym}^{|\omega|}(V)\right)\right)
$$

one for each type of partition. The map $\phi$ restricts to a $\mathbb{Q}\left[\Sigma_{q}\right]$-module map

$$
\phi_{\mathcal{P}\left(\lambda ; L_{1}, L_{2}, \ldots\right)}: \mathbb{Q}\left\{\mathcal{P}\left(\lambda ; L_{1}, L_{2}, \ldots\right)\right\} \longrightarrow M\left(\lambda ; L_{1}, L_{2}, \ldots\right),
$$

and it is enough to show that each of these is an isomorphism.

As $G L(V)$-representations we have a surjection

$$
\bigotimes_{\omega \in \cup_{i} \Omega_{i}}\left(\{\omega\} \otimes V^{\otimes|\omega|}\right)^{\otimes a_{\omega}} \longrightarrow \bigotimes_{\omega \in \cup_{i} \Omega_{i}} \operatorname{Sym}^{a_{\omega}}\left(\{\omega\} \otimes \operatorname{Sym}^{|\omega|}(V)\right)
$$

which is split by the standard symmetrisers. Furthermore if we choose a $(P, \ell) \in$ $\mathcal{P}\left(\lambda ; L_{1}, L_{2}, \ldots\right)$ then we obtain an isomorphism

$$
\begin{aligned}
V^{\otimes q} \stackrel{\sim}{\longrightarrow} \bigotimes_{\omega \in \cup_{i} \Omega_{i}}\left(\{\omega\} \otimes V^{\otimes|\omega|}\right)^{\otimes a_{\omega}} \\
v_{1} \otimes \cdots \otimes v_{q} \longmapsto \bigotimes_{X \subset P}\left(\ell_{X} \otimes \bigotimes_{i \in X} v_{i}\right)
\end{aligned}
$$

(where these terms must be suitably permuted to be put in the right form).

In total this identifies $M\left(\lambda ; L_{1}, L_{2}, \ldots\right)$ with a summand of $\operatorname{Hom}_{G L(V)}\left(V^{\otimes q}, V^{\otimes q}\right)$, which by the first fundamental theorem of invariant theory for $G L(V)$ is a free left $\mathbb{Q}\left[\Sigma_{q}\right]$-module generated by the identity map of $V^{\otimes q}$ (as long as $\operatorname{dim}_{\mathbb{Q}} V \geq q$ ). Thus the $\mathbb{Q}\left[\Sigma_{q}\right]$-module $M\left(\lambda ; L_{1}, L_{2}, \ldots\right)$ may be identified with the cyclic left submodule of $\mathbb{Q}\left[\Sigma_{q}\right]$ generated by the idempotent $\gamma:=\frac{1}{|S|} \sum_{\sigma \in S} \sigma$, where $S \leq \Sigma_{q}$ is the stabiliser of $(P, \ell) \in \mathcal{P}_{q}\left(\Omega_{1}, \Omega_{2}, \ldots\right)$.

Under this identification we have $\phi_{\mathcal{P}\left(\lambda ; L_{1}, L_{2}, \ldots\right)}(P, \ell)=\gamma$, so $\phi_{\mathcal{P}\left(\lambda ; L_{1}, L_{2}, \ldots\right)}$ is surjective. On the other hand, we claim that the submodule $\mathbb{Q}\left[\Sigma_{q}\right] \cdot \gamma \leq \mathbb{Q}\left[\Sigma_{q}\right]$ is isomorphic to $\mathbb{Q}\left[\Sigma_{q} / S\right]$, and hence has dimension the size of the orbit $\mathcal{P}\left(\lambda ; L_{1}, L_{2}, \ldots\right)$. This implies that $\phi_{\mathcal{P}\left(\lambda ; L_{1}, L_{2}, \ldots\right)}$ is an isomorphism. To prove the claim, note that the surjective module map $\mathbb{Q}\left[\Sigma_{q}\right] \cdot \gamma \oplus \mathbb{Q}\left[\Sigma_{q}\right] \cdot(1-\gamma)=\mathbb{Q}\left[\Sigma_{q}\right] \rightarrow \mathbb{Q}\left[\Sigma_{q} / S\right]$ sends $1-\gamma$ to 0 , so gives a surjection $\mathbb{Q}\left[\Sigma_{q}\right] \cdot \gamma \rightarrow \mathbb{Q}\left[\Sigma_{q} / S\right]$. On the other hand the module map

$$
\sum a_{g} g S \mapsto \sum a_{g} g \cdot \gamma: \mathbb{Q}\left[\Sigma_{q} / S\right] \longrightarrow \mathbb{Q}\left[\Sigma_{q}\right] \cdot \gamma
$$

is well-defined and surjective. 


\subsection{Proof of Theorem A}

We have already proved Theorem A (i) in Corollary 3.6. For Theorem A (ii), first choose a functorial model for Eilenberg-MacLane spaces $K(-, n)$, then fix a finitedimensional $\mathbb{Q}$-vector space $V$ and consider the fibration

$$
K\left(H^{*} \otimes_{\mathbb{Z}} V^{*}, 1\right) \simeq \operatorname{map}_{*}\left(\vee^{n} S^{1}, K\left(V^{*}, 2\right)\right) \longrightarrow \mathcal{G}_{n}^{1}\left(K\left(V^{*}, 2\right)\right) \longrightarrow \mathcal{G}_{n}^{1}(*) \simeq B \operatorname{Aut}\left(F_{n}\right)
$$

and its associated Serre spectral sequence with $\mathbb{Q}$-coefficients

$$
E_{2}^{p, q}:=H^{p}\left(\operatorname{Aut}\left(F_{n}\right) ; \wedge^{q}\left(H_{\mathbb{Q}} \otimes V\right)\right) \Longrightarrow H^{p+q}\left(\mathcal{G}_{n}^{1}\left(K\left(V^{*}, 2\right)\right) ; \mathbb{Q}\right) .
$$

The action of $G L(V)$ on $K\left(V^{*}, 2\right)$, and hence on the fibration above, make this into a spectral sequence of $\mathbb{Q}[G L(V)]$-modules.

The proof of the following key lemma is close to an argument communicated to the author by Søren Galatius to prove [23, Conjecture C]. It was his argument that led us to think along the lines necessary to prove Theorem A.

Lemma 5.2 The spectral sequence (5.1) collapses.

Proof The action of the scalars $\mathbb{Q}^{\times} \leq G L(V)$ on $V$ makes it a spectral sequence of $\mathbb{Q}\left[\mathbb{Q}^{\times}\right]$-modules, and the action of $\mathbb{Q}^{\times}$on the $\mathbb{Q}$-vector space $\wedge^{q}\left(H_{\mathbb{Q}} \otimes V\right)$ is with weight $q$, i.e. $u \in \mathbb{Q}^{\times}$acts by scalar multiplication by $u^{q}$. As $\mathbb{Q}$ has characteristic zero, distinct weights make $\mathbb{Q}$ into distinct irreducible $\mathbb{Q}\left[\mathbb{Q}^{\times}\right]$-modules. Thus there can be no $\mathbb{Q}\left[\mathbb{Q}^{\times}\right]$-module maps between different rows of this spectral sequence, so it collapses.

Furthermore, this argument identifies $H^{p}\left(\operatorname{Aut}\left(F_{n}\right) ; \wedge_{\mathbb{Q}}^{q}\left(H_{\mathbb{Q}} \otimes V\right)\right)$ with the subspace $H^{p+q}\left(\mathcal{G}_{n}^{1}\left(K\left(V^{*}, 2\right)\right) ; \mathbb{Q}\right)^{(q)}$ of $H^{p+q}\left(\mathcal{G}_{n}^{1}\left(K\left(V^{*}, 2\right)\right) ; \mathbb{Q}\right)$ on which $\mathbb{Q}^{\times}$acts with weight $q$.

Lemma 5.3 If $2(p+q) \leq n-3$ then $H^{p+q}\left(\mathcal{G}_{n}^{1}\left(K\left(V^{*}, 2\right)\right) \text {; } \mathbb{Q}\right)^{(q)}$ is zero unless $p=q$, in which case it is isomorphic to the degree $2 q$ part of $\operatorname{Sym}^{*}\left(\operatorname{Sym}^{*>0}(V[2])\right)$.

Proof By Theorems 4.1 and 4.2, the map

$$
\tau_{n}^{1}: \mathcal{G}_{n}^{1}\left(K\left(V^{*}, 2\right)\right) \longrightarrow Q_{1-n}\left(K\left(V^{*}, 2\right)_{+}\right)
$$

is an isomorphism on cohomology in degrees $2 * \leq n-3$. With coefficients of characteristic zero, $\widetilde{H}^{*}\left(K\left(V^{*}, 2\right) ; \mathbb{Q}\right) \cong \operatorname{Sym}^{*>0}(V[2])$, and taking the free infinite loop space has the effect of forming the free graded-commutative algebra, so in this case the symmetric algebra. The action of $\mathbb{Q}^{*}$ on $\operatorname{Sym}^{*}\left(\operatorname{Sym}^{*>0}(V[2])\right)$ is with weight $q$ precisely in degree $2 q$.

Corollary 5.4 If $2(p+q) \leq n-3$ then $H^{p}\left(\operatorname{Aut}\left(F_{n}\right) ; \wedge_{\mathbb{Q}}^{q}\left(H_{\mathbb{Q}} \otimes_{\mathbb{Q}} V\right)\right)$ is zero unless $p=q$, in which case it is isomorphic to the degree $2 q$ part of $\operatorname{Sym}^{*}\left(\operatorname{Sym}^{*>0}(V[2])\right)$. 
This proves the vanishing part of Theorem A (ii). We shall now use the fact that the identifications made so far are functorial in $V$, and so are in particular $G L(V)$ equivariant and can be decomposed into irreducible $G L(V)$-modules. The following is a standard consequence of Schur-Weyl duality, but we explain its proof anyway.

Lemma 5.5 As a $G L\left(H_{\mathbb{Q}}\right) \times G L(V)$-representation,

$$
\wedge_{\mathbb{Q}}^{q}\left(H_{\mathbb{Q}} \otimes V\right) \cong \bigoplus_{|\lambda|=q} S_{\lambda}\left(H_{\mathbb{Q}}\right) \otimes S_{\lambda^{\prime}}(V)
$$

where $\lambda^{\prime}$ denotes the conjugate (i.e. transpose) Young diagram to $\lambda$.

Proof The left-hand side is the $\Sigma_{q}$-invariants in the $G L\left(H_{\mathbb{Q}}\right) \times G L(V) \times \Sigma_{q}$-module $\left(H_{\mathbb{Q}} \otimes_{\mathbb{Q}} V\right)^{\otimes q} \otimes \mathbb{Q}^{-} \cong H_{\mathbb{Q}}^{\otimes q} \otimes V^{\otimes q} \otimes \mathbb{Q}^{-}$. By our definition of Schur functors we have an isomorphism of $G L(V) \times \Sigma_{q}$-modules

$$
V^{\otimes q} \cong \bigoplus_{\lambda \vdash q} S_{\lambda}(V) \otimes S^{\lambda}
$$

where $S^{\lambda}$ is the Specht module. Similarly $H_{\mathbb{Q}}^{\otimes q} \cong \bigoplus_{\mu \vdash q} S_{\mu}\left(H_{\mathbb{Q}}\right) \otimes S^{\mu}$, so tensoring them together and taking $\Sigma_{q}$-invariants, using $\left(S^{\mu} \otimes S^{\lambda} \otimes \mathbb{Q}^{-}\right)^{\Sigma_{q}}=\mathbb{Q}^{\delta} \mu \lambda^{\prime}$, the result follows.

Putting the above together, we have an isomorphism of $G L(V)$-modules

$$
\bigoplus_{|\lambda|=q} H^{q}\left(\operatorname{Aut}\left(F_{n}\right) ; S_{\lambda}\left(H_{\mathbb{Q}}\right)\right) \otimes S_{\lambda^{\prime}}(V) \cong\left[\operatorname{Sym}^{*}\left(\operatorname{Sym}^{*>0}(V[2])\right)\right]_{2 q}
$$

Choosing $V$ to be at least $q$-dimensional, the $S_{\mu}(V)$ are then distinct non-zero irreducible $G L(V)$-modules, so by Schur's lemma applying $\operatorname{Hom}_{G L(V)}\left(S_{\lambda^{\prime}}(V),-\right)$ gives

$$
H^{q}\left(\operatorname{Aut}\left(F_{n}\right) ; S_{\lambda}\left(H_{\mathbb{Q}}\right)\right) \cong \operatorname{Hom}_{G L(V)}\left(S_{\lambda^{\prime}}(V), \operatorname{Sym}^{*}\left(\operatorname{Sym}^{*>0}(V)\right)\right)
$$

as long as $n \geq 2 q+3$. Using $H_{\mathbb{Q}}^{\otimes q}=\bigoplus_{|\lambda|=q} S_{\lambda}\left(H_{\mathbb{Q}}\right) \otimes S^{\lambda}$, we obtain

$$
H^{q}\left(\operatorname{Aut}\left(F_{n}\right) ; H_{\mathbb{Q}}^{\otimes q}\right) \cong \operatorname{Hom}_{G L(V)}\left(\bigoplus_{|\lambda|=q} S_{\lambda^{\prime}}(V) \otimes S^{\lambda}, \operatorname{Sym}^{*}\left(\operatorname{Sym}^{*>0}(V)\right)\right)
$$

and using that $S^{\lambda^{\prime}} \cong S^{\lambda} \otimes \mathbb{Q}^{-}$we can write the right-hand side as

$$
\operatorname{Hom}_{G L(V)}\left(V^{\otimes q}, \operatorname{Sym}^{*}\left(\operatorname{Sym}^{*>0}(V)\right)\right) \otimes \mathbb{Q}^{-} .
$$

Along with Proposition 5.1 this finishes the proof of Theorem A. 
Remark 5.6 We could have used $K\left(V^{*}, 3\right)$ instead of $K\left(V^{*}, 2\right)$. In this case the argument goes through, the analogue of Lemma 5.5 is $\operatorname{Sym}^{q}\left(H_{\mathbb{Q}} \otimes V\right) \cong \bigoplus_{|\lambda|=q} S_{\lambda}\left(H_{\mathbb{Q}}\right) \otimes$ $S_{\lambda}(V)$, and the result obtained is

$$
H^{q}\left(\operatorname{Aut}\left(F_{n}\right) ; S_{\lambda}\left(H_{\mathbb{Q}}\right)\right) \cong \operatorname{Hom}_{G L(V)}\left(S_{\lambda}(V), S^{*}\left(\wedge^{*>0}(V)\right)\right)
$$

whenever $\operatorname{dim}_{\mathbb{Q}} V \geq q$ and $n \geq 2 q+3$, where $S^{*}(-)$ denotes the free gradedcommutative algebra.

A consequence of this is that the multiplicity of $S_{\lambda}(V)$ in $S^{*}\left(\wedge^{*>0}(V)\right)$ is the same as the multiplicity of $S_{\lambda^{\prime}}(V)$ in $\operatorname{Sym}^{*}\left(\operatorname{Sym}^{*>0}(V)\right)$, which does not seem obvious to the author.

\subsection{Proof of Theorem B}

We will make use of the following lemma, which follows from Kawazumi [18, Theorem 7.1]. It also follows from general principles: the Becker-Gottlieb transfer with local coefficients.

Lemma 5.7 For any $\mathbb{Z}\left[\frac{1}{n-1}\right]\left[\operatorname{Out}\left(F_{n}\right)\right]$-module $M$, the map

$$
H^{*}\left(\operatorname{Out}\left(F_{n}\right) ; M\right) \longrightarrow H^{*}\left(\operatorname{Aut}\left(F_{n}\right) ; M\right)
$$

is split injective, and

$$
H^{*}\left(\operatorname{Aut}\left(F_{n}\right) ; M\right) \cong H^{*}\left(\operatorname{Out}\left(F_{n}\right) ; M\right) \oplus H^{*-1}\left(\operatorname{Out}\left(F_{n}\right) ; H^{*} \otimes M\right) .
$$

Theorem B (i) and the first part of Theorem B (ii) follows immediately from this lemma, as it implies that $H^{i}\left(\operatorname{Out}\left(F_{n}\right) ; S_{\lambda}\left(H_{\mathbb{Q}}^{*}\right)\right)$ and $H^{i}\left(\operatorname{Out}\left(F_{n}\right) ; S_{\lambda}\left(H_{\mathbb{Q}}\right)\right)$ are summands of $H^{i}\left(\operatorname{Aut}\left(F_{n}\right) ; S_{\lambda}\left(H_{\mathbb{Q}}^{*}\right)\right)$ and $H^{i}\left(\operatorname{Aut}\left(F_{n}\right) ; S_{\lambda}\left(H_{\mathbb{Q}}\right)\right)$ respectively, so vanish under the stated assumptions.

It remains to prove the second part of Theorem B (ii). To do this, we again consider a finite-dimensional $\mathbb{Q}$-vector space $V$ and consider the diagram

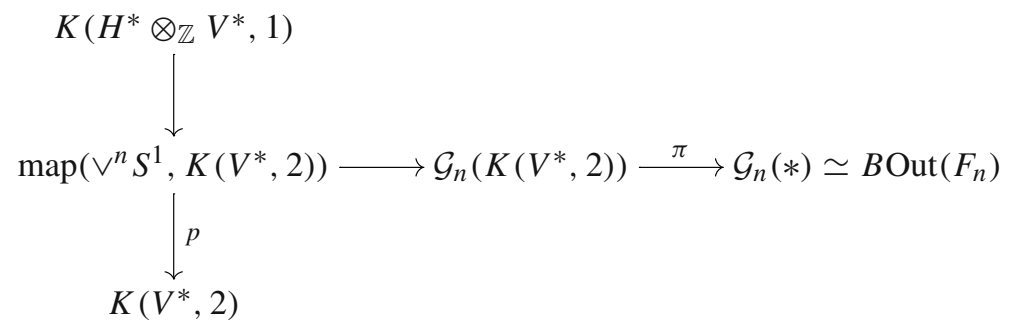

where the row is a (split) fibration and the column is a (trivial) fibration. This gives a spectral sequence

$$
H^{*}\left(\operatorname{Out}\left(F_{n}\right) ; \wedge^{*}\left(H_{\mathbb{Q}} \otimes V[1]\right)\right) \otimes \operatorname{Sym}^{*}(V[2]) \Longrightarrow H^{*}\left(\mathcal{G}_{n}\left(K\left(V^{*}, 2\right)\right) ; \mathbb{Q}\right) .
$$


Rather than a weight argument, which is no longer conclusive, we will deduce the degeneration of this spectral sequence from the vanishing results already established, namely that $H^{i}\left(\operatorname{Out}\left(F_{n}\right) ; S_{\lambda}\left(H_{\mathbb{Q}}\right)\right)=0$ in degrees $2 i \leq n-|\lambda|-3$ if $i \neq|\lambda|$. By Lemma 5.5 this implies that $H^{p}\left(\operatorname{Out}\left(F_{n}\right) ; \wedge^{q}\left(H_{\mathbb{Q}} \otimes V[1]\right)\right)=0$ as long as $2 p \leq$ $n-q-3$ and $p \neq q$, so in bidegrees $(p, q)$ such that $2 p \leq n-q-3$ the $E^{2}$-page of the spectral sequence (5.2) is

$$
\bigoplus_{\substack{a \geq 0 \\ b \geq 0}} H^{a}\left(\operatorname{Out}\left(F_{n}\right) ; \wedge^{a}\left(H_{\mathbb{Q}} \otimes V[1]\right)\right) \otimes \operatorname{Sym}^{b}(V[2])
$$

where the $(a, b)$ th summand lies in $E_{a, a+2 b}^{2}$. In particular, all summands in this range lie in even total degree, and so there can be no differentials.

Lemma 5.8 There is an isomorphism of graded $G L(V)$-representations

$$
\bigoplus_{\lambda} H^{*}\left(\operatorname{Out}\left(F_{\infty}\right) ; S_{\lambda}\left(H_{\mathbb{Q}}\right)\right) \otimes S_{\lambda^{\prime}}(V[1]) \cong \operatorname{Sym}^{*}\left(\operatorname{Sym}^{*>1}(V[2])\right),
$$

and $\operatorname{dim}_{\mathbb{Q}} H^{|\lambda|}\left(\operatorname{Out}\left(F_{n}\right) ; S_{\lambda}\left(H_{\mathbb{Q}}\right)\right)$ is independent of $n$ for $n \geq 4|\lambda|+3$.

Proof Writing $\wedge^{a}\left(H_{\mathbb{Q}} \otimes_{\mathbb{Q}} V[1]\right) \cong \bigoplus_{|\mu|=a} S_{\mu}\left(H_{\mathbb{Q}}\right) \otimes S_{\mu^{\prime}}(V[1])$ using Lemma 5.5, and then identifying the abutment of the spectral sequence (5.2) as in Lemma 5.3, in total degree $2 r$ we obtain

$$
\begin{aligned}
& \bigoplus \bigoplus_{a+b=r|\mu|=a} \bigoplus^{a}\left(\operatorname{Out}\left(F_{n}\right) ; S_{\mu}\left(H_{\mathbb{Q}}\right)\right) \otimes S_{\mu^{\prime}}(V) \otimes \operatorname{Sym}^{b}(V) \\
& \cong\left[\operatorname{Sym}^{*}\left(\operatorname{Sym}^{*>0}(V[2])\right)\right]_{2 r}
\end{aligned}
$$

as long as $4 r \leq n-3$.

The right-hand side is independent of $n$. For a partition $\lambda \vdash r$ we find that

$$
\sum_{\substack{a+b=r \\|\mu|=a}} \operatorname{dim}_{\mathbb{Q}} H^{a}\left(\operatorname{Out}\left(F_{n}\right) ; S_{\mu}\left(H_{\mathbb{Q}}\right)\right) \cdot \operatorname{dim}_{\mathbb{Q}} \operatorname{Hom}_{G L(V)}\left(S_{\lambda^{\prime}}(V), S_{\mu^{\prime}}(V) \otimes \operatorname{Sym}^{b}(V)\right)
$$

is independent of $n$ as long as $n \geq 4|\mu|+3$. By induction we may assume that all terms with $|\mu|<r$ are also independent of $n$, leaving just the terms with $b=0$, which gives $\operatorname{dim}_{\mathbb{Q}} H^{|\lambda|}\left(\operatorname{Out}\left(F_{n}\right) ; S_{\lambda}\left(H_{\mathbb{Q}}\right)\right)$ by Schur's lemma. This proves the second part.

Passing to the limit $n \rightarrow \infty$ and summing over all terms above gives an isomorphism of graded $G L(V)$-representations

$$
\bigoplus_{\lambda} H^{*}\left(\operatorname{Out}\left(F_{\infty}\right) ; S_{\lambda}\left(H_{\mathbb{Q}}\right)\right) \otimes S_{\lambda^{\prime}}(V[1]) \otimes \operatorname{Sym}^{*}(V[2]) \cong \operatorname{Sym}^{*}\left(\operatorname{Sym}^{*>0}(V[2])\right) \text {. }
$$

Because the graded $G L(V)$-representation

$$
\operatorname{Sym}^{*}(V[2])=\mathbb{Q} \oplus \operatorname{Sym}^{1}(V[2]) \oplus \operatorname{Sym}^{2}(V[2]) \oplus \cdots
$$


is the trivial representation in grading zero, the isomorphism in the lemma can be established by induction on degree similarly to the argument above. More conceptually, the graded virtual representation $\operatorname{Sym}^{*}(V)$ is invertible under $\otimes$, as it is the trivial representation in grading zero, and cancelling it from both sides gives the required isomorphism.

Proceeding as in the proof of Theorem A, we obtain

$$
H^{q}\left(\operatorname{Out}\left(F_{n}\right) ; H_{\mathbb{Q}}^{\otimes q}\right) \cong \operatorname{Hom}_{G L(V)}\left(V^{\otimes q}, \operatorname{Sym}^{*}\left(\operatorname{Sym}^{*>1}(V)\right)\right) \otimes \mathbb{Q}^{-}
$$

from which Proposition 5.1 implies Theorem B.

\subsection{Proof of Theorem C}

By Remark 2 after Theorem 4.2.2 of [21], there is an identity

$$
\bigoplus_{\lambda} v^{\infty}(\lambda) S_{\lambda}(V) \cong \operatorname{Sym}^{*}\left(\operatorname{Sym}^{*>1}(V)\right)
$$

of $G L(V)$-modules. Compare with Lemma 5.8 for the first statement; the second statement follows from this and (1.1).

\section{Integral and torsion calculations}

The general technique we have been using is not confined to rational coefficients. To give an example of how it may be used more generally, we shall now develop a "tame" strengthening of Theorem A, namely Theorem E (ii). Let us first explain how Theorem E (ii) implies Theorem E (i).

Proof of Theorem $E(i)$ By Theorem A we have that $H^{1}\left(\operatorname{Aut}\left(F_{\infty}\right) ; H_{\mathbb{Q}}\right)=\mathbb{Q}$ and all other rational cohomology groups vanish. By Theorem $\mathrm{E}$ (ii), for each prime number $p$, $H^{*}\left(\operatorname{Aut}\left(F_{\infty}\right) ; H \otimes \mathbb{Z}_{(p)}\right)$ is a free $H^{*}\left(\operatorname{Aut}\left(F_{\infty}\right) ; \mathbb{Z}_{(p)}\right)$-module, so by the calculation above is a free module on a single generator in degree 1 . Hence $H^{1}\left(\operatorname{Aut}\left(F_{n}\right) ; H\right)$ is $\mathbb{Z}$, as it becomes a free $\mathbb{Z}_{(p)}$-module of rank 1 when localised at any prime $p$. Choosing a generator for this group gives a map

$$
H^{*}\left(\operatorname{Aut}\left(F_{n}\right) ; \mathbb{Z}\right) \longrightarrow H^{*+1}\left(\operatorname{Aut}\left(F_{n}\right) ; H\right)
$$

which must be an isomorphism, as it is so when localised at every prime.

In order to prove Theorem E (ii) we first revisit some of the techniques we have used earlier and develop them in the $p$-local rather than rational setting.

Let $q$ be fixed and $p$ be a prime number such that $p>q$. Recall that to a partition $\lambda \vdash q$ and a tableau $T$ of shape $\lambda$ there is an associated Young symmetriser $c_{T} \in$ $\mathbb{Z}\left[\Sigma_{q}\right]$, which satisfies $c_{T}^{2}=\frac{q !}{\operatorname{dim}_{\mathbb{Q}} S^{\lambda}} c_{T}$. As $q$ ! is a $p$-local unit we may form the 
element $e_{T}:=\frac{\operatorname{dim}_{\mathbb{Q}} S^{\lambda}}{q !} c_{T} \in \mathbb{Z}_{(p)}\left[\Sigma_{q}\right]$ and this is an idempotent. If $g \in \Sigma_{q}$ then $e_{g T}=g e_{T} g^{-1}$, so there is a conjugacy class of idempotent associated to each $\lambda$. We write $e_{\lambda}$ for the idempotent associated to the canonical tableau for $\lambda$. The Specht module is defined by $S^{\lambda}:=\mathbb{Q}\left[\Sigma_{q}\right] \cdot e_{\lambda}$, and by analogy we define the $p$-local Specht module by $S_{(p)}^{\lambda}:=\mathbb{Z}_{(p)}\left[\Sigma_{q}\right] \cdot e_{\lambda}$. It is a free $\mathbb{Z}_{(p)}$-module of rank $\operatorname{dim}_{\mathbb{Q}} S^{\lambda}$, and is indecomposable as a $\mathbb{Z}_{(p)}\left[\Sigma_{q}\right]$-module (as $S_{(p)}^{\lambda} \otimes \mathbb{Q}=S^{\lambda}$ ). These idempotents satisfy $e_{T} \cdot e_{S}=0$ if $T$ and $S$ are tableaux of different shapes. Furthermore we have a decomposition into primitive idempotents

$$
1=\sum_{\lambda \vdash q \text { standard tableaux }} \sum_{\substack{T \text { of shape } \lambda \\ \text { s. }}} e_{T} \in \mathbb{Z}_{(p)}\left[\Sigma_{q}\right]
$$

If $M$ is a $\mathbb{Z}_{(p)}$-module then $M^{\otimes q}$ is a $\mathbb{Z}_{(p)}\left[\Sigma_{q}\right]$-module, and we define the $p$-local Schur functor by $S_{\lambda}(M):=e_{\lambda}\left(M^{\otimes q}\right)$. There is then a natural map

$$
\phi: \bigoplus_{\lambda \vdash q} S_{(p)}^{\lambda} \otimes_{\mathbb{Z}_{(p)}} S_{\lambda}(M) \longrightarrow M^{\otimes q}
$$

of $\mathbb{Z}_{(p)}\left[\Sigma_{q}\right]$-modules given by $\left(x \cdot e_{\lambda}\right) \otimes e_{\lambda}\left(m_{1} \otimes \cdots \otimes m_{q}\right) \mapsto x \cdot e_{\lambda}\left(m_{1} \otimes \cdots \otimes m_{q}\right)$. For an inverse, define

$$
\psi\left(m_{1} \otimes \cdots \otimes m_{q}\right)=\sum_{\lambda \vdash q} \sum_{\substack{\text { standard tableaux } \\ T \text { of shape } \lambda}}\left(g_{T} \cdot e_{\lambda}\right) \otimes e_{\lambda}\left(g_{T}^{-1}\left(m_{1} \otimes \cdots \otimes m_{q}\right)\right)
$$

where for a tableau $T$ of shape $\lambda$ we write $e_{T}=g_{T} e_{\lambda} g_{T}^{-1}$. This establishes the SchurWeyl decomposition in this setting.

We now require a partial analogue of the notion of weights which we used in the proof of Lemma 5.2. For a $\mathbb{Z}_{(p)}$-module $M$ and an integer $t$, write $M(t)$ for the $\mathbb{Z}_{(p)}\left[\mathbb{Z}_{(p)}^{\times}\right]$-module which is the same as a $\mathbb{Z}_{(p)}$-module and on which $u \in \mathbb{Z}_{(p)}^{\times}$acts as scalar multiplication by $u^{t}$; say that it is a module which is pure of weight $t$.

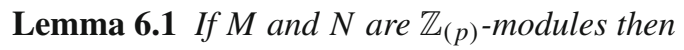

$$
\operatorname{Ext}_{\mathbb{Z}_{(p)}^{*}\left[\mathbb{Z}_{(p)}^{\times}\right]}\left(M(t), N\left(t^{\prime}\right)\right)=0
$$

if $0<\left|t-t^{\prime}\right|<p-1$.

Proof By the natural isomorphisms $X(t) \otimes_{\mathbb{Z}_{(p)}\left[\mathbb{Z}_{(p)}^{\times}\right]} \mathbb{Z}_{(p)}(s) \cong X(t+s)$ it is enough to establish the lemma for $t^{\prime}=0$. In this case consider the functor

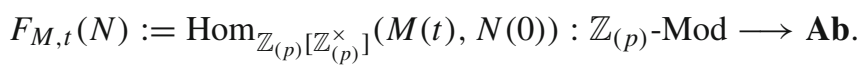


When $M=\mathbb{Z}_{(p)}$ this satisfies

$$
F_{\mathbb{Z}_{(p)}, t}(N)=\left\{x \in N \mid\left(u^{t}-1\right) x=0 \text { for all } u \in \mathbb{Z}_{(p)}^{\times}\right\} .
$$

By [1, Lemma 2.12] the gcd of the numbers $k^{t}-1$ over all integers $k$ coprime to $p$ is itself coprime to $p$ as long as $0<|t|<p-1$, and hence $F_{\mathbb{Z}_{(p)}, t}(N)=0$ under this assumption on $t$. The functor $N \mapsto N(0)$ is exact, so taking derived functors of $F_{\mathbb{Z}_{(p)}, t}$ shows that the claim in the lemma holds for $M=\mathbb{Z}_{(p)}$, and hence for $M$ any free $\mathbb{Z}_{(p)}$-module. The claim in general follows by resolving $M$ by free modules.

We now give the proof of Theorem $\mathrm{E}$ (ii), which states that under our assumptions on $p$ and $q, H^{*}\left(\operatorname{Aut}\left(F_{\infty}\right) ; S_{\lambda}\left(H_{(p)}\right)\right)$ is a free $H^{*}\left(\operatorname{Aut}\left(F_{\infty}\right) ; \mathbb{Z}_{(p)}\right)$-module.

Proof of Theorem E (ii) Let $k$ be an odd integer, and let $S_{(p)}^{k}$ be a choice of model for the $p$-local $k$-sphere. For each unit $u \in \mathbb{Z}_{(p)}^{\times}$we may find a map $f_{u}: S_{(p)}^{k} \rightarrow S_{(p)}^{k}$ inducing multiplication by $u$ on $H_{k}\left(S_{(p)}^{k} ; \mathbb{Z}\right)$, and these satisfy $f_{u} \circ f_{v} \simeq f_{u \cdot v}$. Set $Y:=\left(S_{(p)}^{k}\right)^{q}$, and consider the space $\mathcal{G}_{n}^{1}(Y)$, the Serre fibration

$$
\operatorname{map}_{*}\left(\vee^{n} S^{1}, Y\right) \longrightarrow \mathcal{G}_{n}^{1}(Y) \longrightarrow \mathcal{G}_{n}^{1}(*) \simeq B \operatorname{Aut}\left(F_{n}\right)
$$

and its associated Serre spectral sequence.

Recall that the $\mathbb{Z}_{(p)}$-cohomology ring of $\Omega S_{(p)}^{k}$ is the divided power algebra $\Gamma_{\mathbb{Z}_{(p)}}^{*}\left(x_{k-1}\right)$ on the class obtained by looping a generator of $H^{k}\left(S^{k} ; \mathbb{Z}_{(p)}\right)$. This identifies the $\mathbb{Z}_{(p)}$-cohomology ring of $\operatorname{map}_{*}\left(\vee^{n} S^{1}, Y\right)$ with $\Gamma_{\mathbb{Z}_{(p)}^{*}}\left(H_{(p)}[k-1]\right)^{\otimes q}$, so, taking the limit $n \rightarrow \infty$, there is a spectral sequence

$$
E_{2}^{*, *}=H^{*}\left(\operatorname{Aut}\left(F_{\infty}\right) ; \Gamma_{\mathbb{Z}_{(p)}^{*}}^{*}\left(H_{(p)}[k-1]\right)^{\otimes q}\right) \Longrightarrow H^{*}\left(Q_{0}\left(S^{0}\right) \times Q Y ; \mathbb{Z}_{(p)}\right) .
$$

It follows from the results of $[5$, p. 40] that the map

$$
S_{\mathbb{Z}_{(p)}^{*}}^{*}\left(\widetilde{H}_{*}\left(Y ; \mathbb{Z}_{(p)}\right)\right) \longrightarrow H_{*}\left(Q Y ; \mathbb{Z}_{(p)}\right)
$$

from the free graded-commutative algebra, induced by the map $Y \rightarrow Q(Y)$, is an isomorphism on homology in degrees $* \leq p k$. By the Künneth theorem we have

$$
H_{*}\left(Y ; \mathbb{Z}_{(p)}\right)=\left(\mathbb{Z}_{(p)}[0] \oplus \mathbb{Z}_{(p)}[k]\right)^{\otimes q}
$$

which is free as a $\mathbb{Z}_{(p)}$-module. This shows that $H^{*}\left(Q Y ; \mathbb{Z}_{(p)}\right)$ is a free $\mathbb{Z}_{(p)}$-module in degrees $* \leq p k$, and shows that it is supported in degrees divisible by $k$. Using Galatius' theorem and the Künneth theorem again we may therefore identify the target of the spectral sequence with $H^{*}\left(\operatorname{Aut}\left(F_{\infty}\right) ; \mathbb{Z}_{(p)}\right) \otimes_{\mathbb{Z}_{(p)}} H^{*}\left(Q Y ; \mathbb{Z}_{(p)}\right)$ in a range of degrees. In total degrees $* \leq p k$ the spectral sequence takes the form

$$
E_{2}^{*, *}=H^{*}\left(\operatorname{Aut}\left(F_{\infty}\right) ; \Gamma_{\mathbb{Z}_{(p)}}^{*}\left(H_{(p)}[k-1]\right)^{\otimes q}\right) \Rightarrow H^{*}\left(\operatorname{Aut}\left(F_{\infty}\right) ; \mathbb{Z}_{(p)}\right) \otimes H^{*}\left(Q Y ; \mathbb{Z}_{(p)}\right)
$$


and is a spectral sequence of $H^{*}\left(\operatorname{Aut}\left(F_{\infty}\right) ; \mathbb{Z}_{(p)}\right)$-modules.

For $u \in \mathbb{Z}_{(p)}^{\times}$the homotopy equivalence $f_{u}^{q}: Y \rightarrow Y$ induces a map of spectral sequences by functoriality, making it into a spectral sequence of $\mathbb{Z}_{(p)}\left[\mathbb{Z}_{(p)}^{\times}\right]$-modules. The induced map on $\Gamma_{\mathbb{Z}_{(p)}}^{i}\left(H_{(p)}[k-1]\right)^{\otimes q}$ is given by scalar multiplication by $u^{i q}$, so the $j(k-1)$ st row of the spectral sequence is pure of weight $j$. Furthermore, the fibration (6.1) has a section so there are no differentials entering the bottom row, and this row splits off the filtration. The rows $(k-1), 2(k-1), \ldots,(p-1)(k-$ 1) have different weights which differ by at most $(p-2)$, so by Lemma 6.1 there are no differentials in total degree $*<p(k-1)$ and the associated filtration of $H^{*}\left(\operatorname{Aut}\left(F_{\infty}\right) ; \mathbb{Z}_{(p)}\right) \otimes H^{*}\left(Q Y ; \mathbb{Z}_{(p)}\right)$ splits as $\mathbb{Z}_{(p)}\left[\mathbb{Z}_{(p)}^{\times}\right]$-modules.

The map induced by $f_{u}^{q}$ on $H^{k j}\left(Q Y ; \mathbb{Z}_{(p)}\right)$ is multiplication by $u^{j}$, so this is pure of weight $j$. It follows that the $q(k-1)$ st row of the spectral sequence may be identified in a range of degrees with $H^{*}\left(\operatorname{Aut}\left(F_{\infty}\right) ; \mathbb{Z}_{(p)}\right) \otimes H^{k q}\left(Q Y ; \mathbb{Z}_{(p)}\right)$ and so is a free $H^{*}\left(\operatorname{Aut}\left(F_{\infty}\right) ; \mathbb{Z}_{(p)}\right)$-module. The $\operatorname{Aut}\left(F_{n}\right)$-module $\Gamma_{\mathbb{Z}_{(p)}}^{*}\left(H_{(p)}[k-1]\right)^{\otimes q}$ contains $\left(H_{(p)}[k-1]\right)^{\otimes q}$ as a summand in degree $q(k-1)$, so contains $S_{\lambda}\left(H_{(p)}\right)$ as a summand in this degree too. Thus the $q(k-1)$ st row of the spectral sequence contains $H^{*}\left(\operatorname{Aut}\left(F_{\infty}\right) ; S_{\lambda}\left(H_{(p)}\right)\right)$ as a summand, so this is a projective $H^{*}\left(\operatorname{Aut}\left(F_{\infty}\right) ; \mathbb{Z}_{(p)}\right)$ module in degrees $*<p(k-1)-q(k-1)=(p-q)(k-1)$, so in all degrees as $p>q$ and $k$ was arbitrary. Finally, as $H^{*}\left(\operatorname{Aut}\left(F_{\infty}\right) ; \mathbb{Z}_{(p)}\right)$ is a connected graded algebra over the local ring $\mathbb{Z}_{(p)}$, projective graded modules which are finitely-generated in each degree are free.

Acknowledgements This paper is an update to a 2010 preprint [23] in which I proved, inter alia, Corollary D subject to a sequence of conjectures. Since that time, in joint work with Nathalie Wahl [25] we proved Conjecture A (and I explain in this paper how a version of Conjecture B follows from it), and in 2014 Søren Galatius explained to me a proof of Conjecture C. Thus these conjectural calculations from my 2010 preprint hold. Recent work of Aurélien Djament [8] and Christine Vespa [28] obtains these calculations by very different means. I was motivated by their results to revisit these techniques and to clarify the status of the conjectures from my 2010 preprint. I would like to thank all of the above named for their interest in, and useful comments on, the content of this note.

Open Access This article is distributed under the terms of the Creative Commons Attribution 4.0 International License (http://creativecommons.org/licenses/by/4.0/), which permits unrestricted use, distribution, and reproduction in any medium, provided you give appropriate credit to the original author(s) and the source, provide a link to the Creative Commons license, and indicate if changes were made.

\section{Appendix A: An integral calculation for $\operatorname{Out}\left(F_{n}\right)$}

It seems reasonable to suppose that Theorem 4.2 (ii) holds with integral and not just $\mathbb{Z}\left[\frac{1}{n-1}\right]$-module coefficients, that is, that

Conjecture A The map $\mathcal{G}_{n}^{1}(X) \rightarrow \mathcal{G}_{n}(X)$ induces an isomorphism on homology in degrees $2 * \leq n-3$.

Putting this together with Theorem 4.1, it follows that $\tau_{n}^{0}: \mathcal{G}_{n}(X) \rightarrow Q_{1-n}\left(X_{+}\right)$ is an isomorphism on integral homology in degrees $2 * \leq n-3$. Assuming this conjecture, we may make the following calculation. 
Proposition A.1 We have

$$
\begin{aligned}
& H^{1}\left(\operatorname{Out}\left(F_{n}\right) ; H\right)=0 \quad \text { for } n \geq 7 \\
& H^{2}\left(\operatorname{Out}\left(F_{n}\right) ; H\right)=\mathbb{Z} /(n-1) \quad \text { for } n \geq 9
\end{aligned}
$$

We emphasise that this proposition holds without assuming Conjecture A: it follows from Theorem B that these groups are torsion, Satoh [26] has computed that $H_{1}\left(\operatorname{Out}\left(F_{n}\right) ; H^{*}\right) \cong \mathbb{Z} /(n-1)$ for $n \geq 4$, and one easily computes that $H_{0}\left(\operatorname{Out}\left(F_{n}\right) ; H^{*}\right)=0$. Our purpose here is to give another proof of this proposition using Conjecture A.

This proposition should be contrasted with with a theorem of Bridson and Vogtmann [2, Theorem B], who show that the extension

$$
H=F_{n} / F_{n}^{\prime} \longrightarrow \operatorname{Aut}\left(F_{n}\right) / F_{n}^{\prime} \longrightarrow \operatorname{Out}\left(F_{n}\right)
$$

is non-trivial for all $n \geq 2$, and hence gives a non-trivial class $\zeta \in H^{2}\left(\operatorname{Out}\left(F_{n}\right) ; H\right)$. Our calculation $H^{2}\left(\operatorname{Out}\left(F_{n}\right) ; H\right)=\mathbb{Z} /(n-1)$ along with the result of Bridson and Vogtmann [2] that their class $\zeta$ remains non-trivial in the group $H^{2}\left(\operatorname{Out}\left(F_{n}\right) ; H / r H\right)$ for any $r$ not coprime to $(n-1)$ implies that the class $\zeta$ generates $H^{2}\left(\operatorname{Out}\left(F_{n}\right) ; H\right)$ as long as $n \geq 9$.

Proof of Proposition A.1 Assuming Conjecture A Consider the diagram

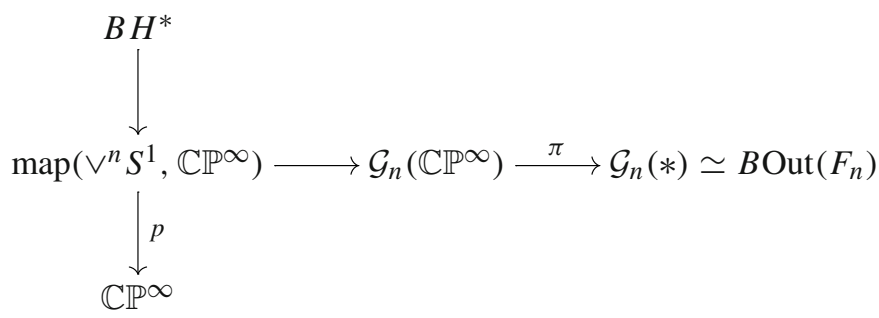

where the row and column are fibrations. Note that $p$ is a trivial fibration and is split via the inclusion $s: \mathbb{C P} \infty \rightarrow \operatorname{map}\left(\vee^{n} S^{1}, \mathbb{C P}^{\infty}\right)$ of the constant maps, and there is an inclusion $\iota: \mathcal{G}_{n}(*) \times \mathbb{C P}^{\infty} \rightarrow \mathcal{G}_{n}\left(\mathbb{C P}^{\infty}\right)$ of the graphs with constant maps to $\mathbb{C P}^{\infty}$. The Leray-Serre spectral sequence for the horizontal fibration is

$$
\bar{E}_{2}^{p, *}:=H^{p}\left(\operatorname{Out}\left(F_{n}\right) ; \wedge^{*} H\right) \otimes \mathbb{Z}[a] \Longrightarrow H^{*}\left(\mathcal{G}_{n}\left(\mathbb{C P}^{\infty}\right) ; \mathbb{Z}\right)
$$

where $a$ is the canonical class in $H^{2}\left(\mathbb{C P}^{\infty} ; \mathbb{Z}\right)$, so has bidegree $(p, q)=(0,2)$.

We first claim that the map

$$
\mathbb{C} \mathbb{P}^{\infty} \stackrel{s}{\longrightarrow} \operatorname{map}\left(\vee^{n} S^{1}, \mathbb{C P}^{\infty}\right) \longrightarrow \mathcal{G}_{n}\left(\mathbb{C P}^{\infty}\right)
$$

has image $(n-1) \mathbb{Z} \subset \mathbb{Z}=H^{2}\left(\mathbb{C P}^{\infty} ; \mathbb{Z}\right)$ on second cohomology. By our conjecture, it is enough to prove this after composing with the map $\tau_{n}^{0}: \mathcal{G}_{n}\left(\mathbb{C P}^{\infty}\right) \rightarrow Q_{0}\left(\mathbb{C P}_{+}^{\infty}\right)$ 
as long as $n \geq 7$. Up to translation of components, this map is given by the BeckerGottlieb transfer $\mathbb{C P}^{\infty} \rightarrow Q_{1-n}\left(\mathbb{C P}^{\infty} \times\left(\vee^{n} S^{1}\right)_{+}\right)$for the trivial graph bundle over $\mathbb{C P}^{\infty}$ composed with projection to $Q_{1-n}\left(\mathbb{C P}_{+}^{\infty}\right)$. By standard properties of the transfer, this is $(1-n)$ times the standard inclusion, which on second cohomology induces multiplication by $(1-n)$, as required.

This describes the edge homomorphism of the spectral sequence (A.1). We now use the homotopy equivalence

$$
Q_{0}\left(\mathbb{C P}_{+}^{\infty}\right) \simeq Q_{0}\left(S^{0}\right) \times Q\left(\mathbb{C P}^{\infty}\right)
$$

and that $S^{2} \rightarrow Q\left(\mathbb{C} \mathbb{P}^{\infty}\right)$ is 3-connected (by the Freudenthal suspension theorem) to describe the cohomology of $Q_{0}\left(\mathbb{C P}_{+}^{\infty}\right)$ in low degrees. Using that $H^{i}\left(Q_{0}\left(S^{0}\right) ; \mathbb{Z}\right)$ is $\mathbb{Z}, 0, \mathbb{Z} / 2, \mathbb{Z} / 2$ for $i=0,1,2,3$, it follows that $H^{i}\left(Q_{0}\left(\mathbb{C P}_{+}^{\infty}\right) ; \mathbb{Z}\right)$ is $\mathbb{Z}, 0, \mathbb{Z} \oplus$ $\mathbb{Z} / 2, \mathbb{Z} / 2$ for $i=0,1,2,3$. As the spectral sequence (A.1) converges to zero for positive Leray filtration in total degree 3 , the differential $d_{2}: \mathbb{Z}=\bar{E}_{2}^{0,2} \rightarrow \bar{E}_{2}^{2,1}$ must be onto (so $\bar{E}_{2}^{2,1}$ is cyclic) and the kernel is $(n-1) \mathbb{Z}$, so $\bar{E}_{2}^{2,1}=H^{2}\left(\operatorname{Out}\left(F_{n}\right) ; H\right) \cong$ $\mathbb{Z} /(n-1)$. On the other hand, in total degree 2 we see $(n-1) \mathbb{Z}=\bar{E}_{\infty}^{0,2}$ and $\mathbb{Z} / 2=\bar{E}_{\infty}^{2,0}$, and it converges to $\mathbb{Z} / 2 \oplus \mathbb{Z}$, so observing the direction of the Leray filtration we see that $H^{1}\left(\operatorname{Out}\left(F_{n}\right) ; H\right)=\bar{E}_{2}^{1,1}=0$.

\section{Appendix B: Mapping class groups of surfaces}

Let $\Gamma_{g}$ denote the mapping class group of a surface $\Sigma_{g}$ of genus $g$, and consider $H_{\mathbb{Q}}:=H_{1}\left(\Sigma_{g} ; \mathbb{Q}\right)$ as a $\Gamma_{g}$-module. In this case Poincaré duality gives $H_{\mathbb{Q}}^{*} \cong H_{\mathbb{Q}}$. Looijenga has already computed $H^{*}\left(\Gamma_{g} ; S_{\lambda}\left(H_{\mathbb{Q}}\right)\right)$ in the stable range [20], but we wish to explain here how the computation may also be performed using the techniques of this paper.

Cohen and Madsen [6] have introduced spaces $\mathcal{S}_{g}(X)$ of surfaces diffeomorphic to $\Sigma_{g}$ equipped with a map to $X$, and have identified the stable (co)homology of these spaces with that of the infinite loop space $\Omega^{\infty}\left(\operatorname{MTSO}(2) \wedge X_{+}\right)$as long as $X$ is simply-connected. Furthermore, there is a fibration sequence

$$
\operatorname{map}\left(\Sigma_{g}, X\right) \longrightarrow \mathcal{S}_{g}(X) \longrightarrow B \Gamma_{g}
$$

so an associated Serre spectral sequence.

Considering $X=K\left(V^{*}, 4\right)$, the argument of Sect. 5.4 goes through without change and identifies

$$
\bigoplus_{\lambda} H^{*}\left(\Gamma_{g} ; S_{\lambda}\left(H_{\mathbb{Q}}\right)\right) \otimes S_{\lambda^{\prime}}(V)[3|\lambda|] \otimes \operatorname{Sym}^{*}(V[2]) \otimes \operatorname{Sym}^{*}(V[4])
$$

with

$$
H^{*}\left(\Omega_{0}^{\infty}\left(\operatorname{MTSO}(2) \wedge K\left(V^{*}, 4\right)_{+}\right) ; \mathbb{Q}\right)=\operatorname{Sym}^{*}\left(\left[W_{*} \otimes \operatorname{Sym}^{*}(V[4])\right]_{>0}\right)
$$


in a range of degrees increasing with $g$, where $W_{*}=H^{*}(\operatorname{MTSO}(2) ; \mathbb{Q})$ is the graded vector space which is $\mathbb{Q}$ in degrees $-2,0,2,4,6, \ldots$ and zero otherwise. This may be written as

$$
\begin{aligned}
\operatorname{Sym}^{*}\left(W_{*>0}\right) & \otimes \operatorname{Sym}^{*}(V[2]) \otimes \operatorname{Sym}^{*}(V[4]) \otimes \operatorname{Sym}^{*}\left(W_{*>0}\right. \\
& \left.\otimes V[4] \oplus W_{*} \otimes \operatorname{Sym}^{*>1}(V[4])\right)
\end{aligned}
$$

and the first term $\operatorname{Sym}^{*}\left(W_{*>0}\right)$ is $H^{*}\left(\Gamma_{g} ; \mathbb{Q}\right)$ in the stable range, so we obtain

$$
\begin{aligned}
& \bigoplus_{\lambda} H^{*}\left(\Gamma_{g} ; S_{\lambda}\left(H_{\mathbb{Q}}\right)\right) \otimes S_{\lambda^{\prime}}(V)[3|\lambda|] \cong H^{*}\left(\Gamma_{g} ; \mathbb{Q}\right) \otimes \operatorname{Sym}^{*}\left(W_{*>0} \otimes V[4]\right. \\
& \left.\bigoplus W_{*} \otimes \operatorname{Sym}^{*>1}(V[4])\right)
\end{aligned}
$$

in a range of degrees increasing with $g$.

It follows that each $H^{*}\left(\Gamma_{g} ; S_{\lambda}\left(H_{\mathbb{Q}}\right)\right)$ is a free $H^{*}\left(\Gamma_{g} ; \mathbb{Q}\right)$-module in the stable range. To describe the space of module generators we can proceed as in the proof of Theorem A, and hence identify $H^{*}\left(\Gamma_{g} ; H_{\mathbb{Q}}^{\otimes q}\right)$ as a graded $\Sigma_{q}$-module with

$$
\begin{aligned}
& H^{*}\left(\Gamma_{g} ; \mathbb{Q}\right) \otimes \operatorname{Hom}_{G L(V)}\left(V^{\otimes q}, \operatorname{Sym}^{*}\left(W_{*>0} \otimes V[4]\right.\right. \\
& \left.\left.\oplus W_{*} \otimes \operatorname{Sym}^{*>1}(V[4])\right)\right)[-3 q] \otimes \mathbb{Q}^{-} .
\end{aligned}
$$

Proposition 5.1 identifies the space of $G L(V)$-module homomorphisms with the permutation module on the set of the following data: a partition $P$ of $\{1,2, \ldots, q\}$, a labeling of each part of size 1 in the set $\left\{x_{2}, x_{4}, x_{6}, \ldots\right\}$, and a labeling of each part of size $>1$ in the set $\left\{x_{-2}, x_{0}, x_{2}, x_{4}, x_{6}, \ldots\right\}$. Such a datum is given grading $q$ plus the sum of the degrees of the labels (which are given by their subscripts).

Example B.1 When $q=1$ we find that $H^{*}\left(\Gamma_{g} ; H_{\mathbb{Q}}\right)$ is a free $H^{*}\left(\Gamma_{g} ; \mathbb{Q}\right)$-module with generators in degrees $\{3,5,7, \ldots\}$.

When $q=2$ the partition $\{1,2\}$ may be labeled by $\left\{x_{-2}, x_{0}, x_{2}, x_{4}, x_{6}, \ldots\right\}$, and the partition $\{\{1\},\{2\}\}$ may have each part labeled by $\left\{x_{2}, x_{4}, x_{6}, \ldots\right\}$. As a $\Sigma_{2}$-set the action is trivial on the first type of elements, and the second type form trivial orbits

$$
\left\{\left\{1 ; x_{2 i}\right\},\left\{2 ; x_{2 i}\right\}\right\}
$$

and free orbits

$$
\left\{\left\{1 ; x_{2 i}\right\},\left\{2 ; x_{2 j}\right\}\right\},\left\{\left\{1 ; x_{2 j}\right\},\left\{2 ; x_{2 i}\right\}\right\}
$$

with $i \neq j$. Thus $H^{*}\left(\Gamma_{g} ; \wedge^{2}\left(H_{\mathbb{Q}}\right)\right)$ has $H^{*}\left(\Gamma_{g} ; \mathbb{Q}\right)$-module generators given by the multiplicities of the trivial representation in the indicated permutation module, so in degrees $\{0,2,4,6, \ldots ; 6,8,10, \ldots ; 10,12,14, \ldots ; 14,16,18, \ldots ; \ldots\}$. Similarly $H^{*}\left(\Gamma_{g} ; \operatorname{Sym}^{2}\left(H_{\mathbb{Q}}\right)\right)$ has $H^{*}\left(\Gamma_{g} ; \mathbb{Q}\right)$-module generators given by the multiplicities of the sign representation in the indicated permutation module, so in degrees $\{8,10,12, \ldots ; 12,14,16, \ldots ; 16,18,20, \ldots ; \ldots\}$. 
See [11, Theorem G] for a complete calculation in the case of exterior powers, and [24, Proposition 5.2] for a complete calculation in the case of symmetric powers.

By considering the analogous space $\mathcal{S}_{g, 1}(X)$ of surfaces of genus $g$ with one boundary equipped with maps to $X=K\left(V^{*}, 4\right)$ which map the boundary to the basepoint, one identifies $\bigoplus_{\lambda} H^{*}\left(\Gamma_{g, 1} ; S_{\lambda}\left(H_{\mathbb{Q}}\right)\right) \otimes S_{\lambda^{\prime}}(V)[3|\lambda|]$ with

$$
H^{*}\left(\Gamma_{g, 1} ; \mathbb{Q}\right) \otimes \operatorname{Sym}^{*}\left(W_{*>-2} \otimes V[4] \oplus W_{*} \otimes \operatorname{Sym}^{*>0}(V[4])\right)
$$

in a range of degrees increasing with $g$, from which $H^{*}\left(\Gamma_{g, 1} ; H_{\mathbb{Q}}^{\otimes q}\right)$ may be computed as above. There is a similar description in terms of partitions, the only difference being that now pieces of a partition of size 1 may also be labeled by $x_{0}$. For example, one finds that $H^{*}\left(\Gamma_{g, 1} ; H_{\mathbb{Q}}\right)$ is a free $H^{*}\left(\Gamma_{g, 1} ; \mathbb{Q}\right)$-module with generators in degrees $\{1,3,5,7, \ldots\}$. This description of $H^{*}\left(\Gamma_{g, 1} ; H_{\mathbb{Q}}^{\otimes q}\right)$ (in terms of labelled partitions) has previously been obtained by Kawazumi [19], cf. Theorem 1.B of that paper, even with integral as opposed to just rational coefficients.

\section{Appendix C: Tables}

The result of Theorems A and B for $|\lambda| \leq 6$ is compiled in the tables below, which were computed with the SchurRings package for Macaulay2 [14], and checked via Theorem $C$ and the table at the end of Section 4.3 of [21].

Dimensions of $H^{|\lambda|}\left(\operatorname{Aut}\left(F_{n}\right) ; S_{\lambda}\left(H_{\mathbb{Q}}\right)\right)$ for $|\lambda| \leq 6$ and $n \geq 2|\lambda|+3$.

\begin{tabular}{c|c|cc|ccc|ccccc}
\hline() & $(1)$ & $\left(1^{2}\right)$ & $(2)$ & $\left(1^{3}\right)$ & $(21)$ & $(3)$ & $\left(1^{4}\right)$ & $\left(21^{2}\right)$ & $\left(2^{2}\right)$ & $(31)$ & $(4)$ \\
\hline 1 & 1 & 2 & 0 & 3 & 1 & 0 & 5 & 2 & 2 & 0 & 0 \\
\hline
\end{tabular}

\begin{tabular}{|c|c|c|c|c|c|c|c|c|c|c|}
\hline$\left(1^{5}\right)$ & $\left(21^{3}\right)$ & $\left(2^{2} 1\right)$ & $\left(31^{2}\right)$ & (32) & (41) & (5) & & & & \\
\hline 7 & 5 & 4 & 0 & 1 & 0 & 0 & & & & \\
\hline$\left(1^{6}\right)$ & $\left(21^{4}\right)$ & $\left(2^{2} 1^{2}\right)$ & $\left(2^{3}\right)$ & $\left(31^{3}\right)$ & (321) & $\left(3^{2}\right)$ & $\left(41^{2}\right)$ & (42) & (51) & (6) \\
\hline 11 & 8 & 10 & 2 & 1 & 2 & 2 & 0 & 0 & 0 & 0 \\
\hline
\end{tabular}

Dimensions of $H^{|\lambda|}\left(\operatorname{Out}\left(F_{n}\right) ; S_{\lambda}\left(H_{\mathbb{Q}}\right)\right)$ for $|\lambda| \leq 6$ and $n \geq 4|\lambda|+3$.

\begin{tabular}{|c|c|c|c|c|c|c|c|c|c|c|}
\hline () & (1) & $\begin{array}{ll}(11) & (2)\end{array}$ & (111) & (21) & (3) & (1111) & (211) & (22) & (31) & (4) \\
\hline 1 & 0 & 1 & 1 & 0 & 0 & 2 & 0 & 1 & 0 & 0 \\
\hline \multicolumn{2}{|c|}{ (11111) } & (2111) & (221) & (311) & (32) & (41) & (5) & & & \\
\hline \multicolumn{2}{|c|}{2} & 1 & 1 & 0 & 0 & 0 & 0 & & & \\
\hline$\left(1^{6}\right.$ & (2) & $\left(2^{2} 1^{2}\right)$ & $\left(2^{3}\right)$ & $\left(31^{3}\right)$ & (321) & $\left(3^{2}\right)$ & $\left(41^{2}\right)$ & (42) & (51) & (6) \\
\hline 4 & & 3 & 0 & 0 & 0 & 1 & 0 & 0 & 0 & 0 \\
\hline
\end{tabular}




\section{References}

1. Adams, J.F.: On the groups $J(X)$. II. Topology 3, 137-171 (1965)

2. Bridson, M.R., Vogtmann, K.: Abelian covers of graphs and maps between outer automorphism groups of free groups. Math. Ann. 353(4), 1069-1102 (2012)

3. Brown, K.S.: Cohomology of groups, volume $\mathbf{8 7}$ of Graduate Texts in Mathematics. Springer-Verlag, New York (1994) (corrected reprint of the 1982 original)

4. Cartan, H., Eilenberg, S.: Homological Algebra. Princeton University Press, Princeton (1956)

5. Cohen, F.R., Lada, T.J., May, J.P.: The homology of iterated loop spaces. Lecture Notes in Mathematics, Vol. 533. Springer-Verlag, Berlin (1976)

6. Cohen, R., Madsen, I.: Surfaces in a background space and the homology of mapping class group. Proc. Symp. Pure Math. 80(1), 43-76 (2009)

7. Cohen, R., Madsen, I.: Stability for closed surfaces in a background space. Homol. Homotopy Appl. 13(2), 301-313 (2011)

8. Djament, A.: Décomposition de Hodge pour l'homologie stable des groupes d'automorphismes des groupes libres. arXiv: 1510.03546 (2015)

9. Djament, A., Vespa, C.: Sur l'homologie des groupes d'automorphismes des groupes libres à coefficients polynomiaux. Comment. Math. Helv. 90(1), 33-58 (2015)

10. Dwyer, W.G.: Twisted homological stability for general linear groups. Ann. Math. (2) 111(2), 239-251 (1980)

11. Ebert, J., Randal-Williams, O.: Stable cohomology of the universal Picard varieties and the extended mapping class group. Doc. Math. 17, 417-450 (2012)

12. Galatius, S.: Stable homology of automorphism groups of free groups. Ann. Math. (2) 173(2), 705-768 (2011)

13. Galatius, S., Randal-Williams, O.: Monoids of moduli spaces of manifolds. Geom. Topol. 14(3), 1243$1302(2010)$

14. Grayson, D.R., Stillman, M.E.: Macaulay2, a software system for research in algebraic geometry. http://www.math.uiuc.edu/Macaulay2

15. Hatcher, A., Vogtmann, K.: Cerf theory for graphs. J. Lond. Math. Soc. (2) 58(3), 633-655 (1998)

16. Hatcher, A., Vogtmann, K.: Homology stability for outer automorphism groups of free groups. Algebr. Geom. Topol 4, 1253-1272 (2004). (electronic)

17. Ivanov, N.V.: On the homology stability for Teichmüller modular groups: closed surfaces and twisted coefficients. In: Mapping class groups and moduli spaces of Riemann surfaces (Göttingen, 1991/Seattle, WA, 1991), volume 150 of Contemp. Math., pp. 149-194. Amer. Math. Soc., Providence, RI (1993)

18. Kawazumi, N.: Cohomological aspects of Magnus expansions. arXiv:math/0505497 (2005)

19. Kawazumi, N.: On the stable cohomology algebra of extended mapping class groups for surfaces. In: Groups of Diffeomorphisms, volume 52 of Adv. Stud. Pure Math., pp. 383-400. Math. Soc. Jpn., Tokyo (2008)

20. Looijenga, E.: Stable cohomology of the mapping class group with symplectic coefficients and of the universal Abel-Jacobi map. J. Algebraic Geom. 5(1), 135-150 (1996)

21. Manivel, L.: Gaussian maps and plethysm. In: Algebraic Geometry (Catania, 1993/Barcelona, 1994), volume 200 of Lecture Notes in Pure and Appl. Math., pp. 91-117. Dekker, New York (1998)

22. Procesi, C.: Lie groups, Universitext. Springer, New York (2007)

23. Randal-Williams, O.: The stable cohomology of automorphisms of free groups with coefficients in the homology representation. arXiv:1012.1433 (2010)

24. Randal-Williams, O.: The space of immersed surfaces in a manifold. Math. Proc. Cambridge Philos. Soc. 154(3), 419-438 (2013)

25. Randal-Williams, O., Wahl, N.: Homological stability for automorphism groups. arXiv:1409.3541 (2014)

26. Satoh, T.: Twisted first homology groups of the automorphism group of a free group. J. Pure Appl. Algebra 204(2), 334-348 (2006)

27. Satoh, T.: Twisted second homology groups of the automorphism group of a free group. J. Pure Appl. Algebra 211(2), 547-565 (2007)

28. Vespa, C.: Extensions between functors from groups. arXiv:1511.03098 (2015) 